\title{
A High-resolution Mosaic of the Neutral Hydrogen in the M81 Triplet
}

\author{
de Blok, W. J. G.
}

2018-09-20

de Blok, W J G , Walter , F , Ferguson, A M N , Bernard , E J , van der Hulst, J M , Neeleman , M , Leroy , A K, Ott , J , Zschaechner , L K, Zwaan , M A , Yun , M S , Langston, G \& Keating , K M 2018 , ' A High-resolution Mosaic of the Neutral Hydrogen in the M81 Triplet ' , Astrophysical Journal , vol. 865 , no. 1 , 26 . https://doi.org/10.3847/1538-4357/aad557

http://hdl.handle.net/10138/299393

https://doi.org/10.3847/1538-4357/aad557

unspecified

publishedVersion

Downloaded from Helda, University of Helsinki institutional repository.

This is an electronic reprint of the original article.

This reprint may differ from the original in pagination and typographic detail.

Please cite the original version. 


\title{
A High-resolution Mosaic of the Neutral Hydrogen in the M81 Triplet
}

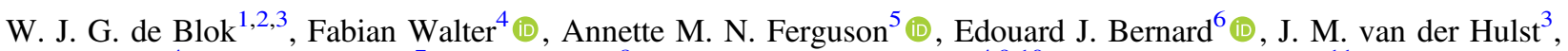 \\ Marcel Neeleman ${ }^{4}$, Adam K. Leroy ${ }^{7}$ (D), Jürgen Ott ${ }^{8}$ (D), Laura K. Zschaechner ${ }^{4,9,10}$ (D), Martin A. Zwaan ${ }^{11}$ (D), Min S. Yun ${ }^{12}$ (D), \\ Glen Langston $^{13}$, and Katie M. Keating ${ }^{14}$ \\ ${ }^{1}$ Netherlands Institute for Radio Astronomy (ASTRON), Postbus 2, 7990 AA Dwingeloo, The Netherlands \\ ${ }^{2}$ Dept. of Astronomy, Univ. of Cape Town, Private Bag X3, Rondebosch 7701, South Africa \\ ${ }^{3}$ Kapteyn Astronomical Institute, University of Groningen, Postbus 800, 9700 AV Groningen, The Netherlands \\ ${ }^{4}$ Max-Planck Institut für Astronomie, Königstuhl 17, D-69117, Heidelberg, Germany \\ ${ }^{5}$ Institute for Astronomy, University of Edinburgh, Royal Observatory, Blackford Hill, Edinburgh EH9 3HJ, UK \\ ${ }^{6}$ Université Côte d'Azur, OCA, CNRS, Lagrange, France \\ ${ }^{7}$ Department of Astronomy, The Ohio State University, 140 W. 18th Avenue, Columbus, OH 43210, USA \\ ${ }^{8}$ National Radio Astronomy Observatory, 1003 Lopezville Road, Socorro, NM 87801, USA \\ ${ }^{9}$ University of Helsinki, P.O. Box 64, Gustaf Hällströmin katu 2a, FI-00014 University of Helsinki, Finland \\ ${ }^{10}$ Finnish Center for Astronomy with ESO, FI-20014 Turun yliopisto, Finland \\ ${ }^{11}$ European Southern Observatory, Karl-Schwarzschild-Str. 2, D-85748 Garching Near Munich, Germany \\ ${ }_{12}$ Department of Astronomy, University of Massachusetts, Amherst, MA 01003, USA \\ ${ }^{13}$ National Science Foundation, Division of Astronomical Sciences, Arlington, VA 22230, USA \\ ${ }^{14}$ Rincon Research Corporation, 101 North Wilmot Road, Suite 101, Tucson, AZ 85711, USA \\ Received 2018 April 16; revised 2018 July 13; accepted 2018 July 19; published 2018 September 18
}

\begin{abstract}
We present a $3^{\circ} \times 3^{\circ}$, 105-pointing, high-resolution neutral hydrogen (H I) mosaic of the M81 galaxy triplet, (including the main galaxies M81, M82, and NGC 3077, as well as dwarf galaxy NGC 2976) obtained with the Very Large Array C and D arrays. This H I synthesis mosaic uniformly covers the entire area and velocity range of the triplet. The observations have a resolution of $\sim 20^{\prime \prime}$ or $\sim 420 \mathrm{pc}$. The data reveal many small-scale anomalous velocity features highlighting the complexity of the interacting M81 triplet. We compare our data with Green Bank Telescope observations of the same area. This comparison provides evidence for the presence of a substantial reservoir of low-column density gas in the northern part of the triplet, probably associated with M82. Such a reservoir is not found in the southern part. We report a number of newly discovered kpc-sized low-mass H I clouds with H I masses of a few times $10^{6} M_{\odot}$. A detailed analysis of their velocity widths show that their dynamical masses are much larger than their baryonic masses, which could indicate the presence of dark matter if the clouds are rotationally supported. However, due to their spatial and kinematical association with $\mathrm{H}$ I tidal features, it is more likely that the velocity widths indicate tidal effects or streaming motions. We do not find any clouds that are not associated with tidal features down to an H I mass limit of a few times $10^{4} M_{\odot}$. We compare the H I column densities with resolved stellar density maps and find a star formation threshold around $3-6 \times 10^{20} \mathrm{~cm}^{-2}$. We investigate the widths of the $\mathrm{HI}$ velocity profiles in the triplet and find that extreme velocity dispersions can be explained by a superposition of multiple components along the line of sight near M81 as well as winds or outflows around M82. The velocity dispersions found are high enough that these processes could explain the linewidths of damped-Ly $\alpha$ absorbers observed at high redshift.
\end{abstract}

Key words: galaxies: fundamental parameters - galaxies: individual (M81, M82, NGC 3077) - galaxies: ISM galaxies: kinematics and dynamics - radio lines: galaxies

\section{Introduction}

The evolution of galaxies is affected by their environment. Galaxy interactions and mergers are probably the most obvious examples of this. These processes can severely alter or completely transform a galaxy's properties. Signs of galaxy interactions are most easily detected in the component of the galaxy that is the most sensitive to them, namely the extended reservoirs of circumgalactic neutral hydrogen (H I) (although evidence for interactions can also be seen in extended stellar envelopes; for an early example see Ferguson et al. 2002).

The M81 triplet (with M81, M82, and NGC 3077 as the main galaxies) is often presented as a prime example of the complexity of interactions, their impact on the circumgalactic medium and, therefore, galaxy evolution (see Yun et al. 1994). The three main galaxies in the triplet each highlight different aspects of galaxy evolution. The inner disk of the grand spiral galaxy M81 seems largely unaffected by the interaction.
Studies of the disk have been instrumental in developing the theory of density waves and formation of (grand) spiral structure (see, e.g., Rots 1975 for an early example). The starburst galaxy M82 is a unique target for studying interactiontriggered star formation feedback processes in essentially all wavelength bands (e.g., $\mathrm{H} \alpha, \mathrm{X}$-rays, dust, $\mathrm{H} \mathrm{I}$, molecular gas; see Walter et al. 2002b; Leroy et al. 2015 and references therein). The third galaxy, NGC 3077, is an optically smooth galaxy with an actively star-forming core, which has been stripped of most of its H I (e.g., Walter et al. 2002a). Much of this $\mathrm{HI}$ is now found immediately to the east of the main galaxy as part of the "Garland" feature (e.g., Yun et al. 1993a; Walter et al. 2011). The triplet is surrounded by at least 20 dwarf galaxies (including a few tidal dwarfs) that together form the greater "M81 group" (e.g., Karachentsev et al. 2002). One of the more prominent of these dwarf galaxies is NGC 2976, an actively star-forming, gas-rich dwarf galaxy. 
Very Large Array (VLA) D-array H I observations of the M81 triplet, obtained by Yun et al. (1994), have played a critical role in shaping our understanding of how interactions between galaxies affect the distribution of the atomic gas (see also Yun et al. 1993a and early work by van der Hulst 1979 and Appleton \& van der Hulst 1988). The 12 pointing ( 2.8 square degrees) mosaic presented in Yun et al. (1994) (which was later extended to 24 pointings ( $\sim 5.6$ square degrees), Yun et al. 2000) demonstrated that the extended HI emission in the triplet is dominated by filamentary structures of many tens of kiloparsecs connecting the main galaxies and containing most of the $\mathrm{HI}$ in the system. These structures are mostly due to the effects of the tidal interactions. No such signs are visible in shallow optical imaging of the triplet. However, recent star count analyses of the triplet, which are sensitive to very low surface brightness emission, have revealed that the stellar component is also affected by the interaction (Okamoto et al. 2015).

Green Bank Telescope (GBT) H I observations (Chynoweth et al. 2008) of the M81 triplet covered a larger area $\left(3^{\circ} \times 3^{\circ}\right)$ down to low column densities, albeit at a spatial resolution $(\sim 10 \mathrm{kpc})$ that is insufficient to resolve the sub-kpc giant atomic complexes that are present in nearby galaxies and that are key to our understanding of star formation (e.g., Bigiel et al. 2008; Leroy et al. 2008). However, the GBT observations clearly demonstrated that there is almost twice as much H I present in the system than detected in the Yun et al. $(1994,2000)$ observations.

Here, we use the dramatic increase in VLA capabilities over the last two decades to completely map at high resolution (spatially and spectrally) the $\mathrm{H}$ I in the entirety of the M81 triplet and its immediate surroundings. We present a 105-pointing (7.6 square degrees out to the $50 \%$ sensitivity level) C-and D-array mosaic, covering the same area as the Chynoweth et al. (2008) GBT observations. These new data form the most complete high-resolution and high-sensitivity census of atomic gas in the M81 triplet so far. Our highest-resolution data set has a resolution of $\sim 24^{\prime \prime}$ (420 pc at $D=3.63 \mathrm{Mpc}$, the distance of the M81 triplet; Karachentsev et al. 2004), or close to a factor of three higher spatial resolution than the earlier 12-pointing data presented in Yun et al. (1994). These earlier data were limited by the capacities of the correlator at the time, with different pointings observed over different, fairly narrow velocity ranges. In our new data, all pointings cover the entire velocity range of the triplet, at a much higher velocity resolution. These data thus form the most complete and comprehensive view to date of the atomic gas in the M81 triplet and its immediate surroundings.

A data set of this size with this level of detail has many applications. Here, apart from presenting the data, we focus on the following aspects. Our $\sim 400 \mathrm{pc}$ resolution observations reach a limiting $\mathrm{HI}$ mass of $\sim 10^{4} M_{\odot}$ and constrain the numbers of individual H I clouds in the group down to very low masses and sizes. This will provide a link to the missing satellite problem, i.e., satellites with clumps of cool H I, but no star formation.

A second important topic is the connection to high-redshift $\mathrm{HI}$ through measurements of the $\mathrm{HI}$ probability distribution function. Linking high-redshift $\mathrm{H}$ I absorption measurements to local emission properties is important as our high- $z \mathrm{HI}$ knowledge is based on absorption measurements. If the M81 triplet were by chance observed in the foreground against a high-redshift quasar, it would be classified as either a Lyman limit system or a damped Ly $\alpha$ absorber (DLA), depending on where exactly the quasar's sightline would appear through the H I distribution. Our covered area measures about
$0.2 \mathrm{Mpc} \times 0.2 \mathrm{Mpc}$, large enough to include typical impact parameters between quasars and DLAs at high redshift (e.g., Rahmati \& Schaye 2014). We can therefore directly compare the H I linewidths in the triplet with those seen in DLA systems at high redshift.

Finally, we address the link between star formation and $\mathrm{H}$ I in the triplet. Empirical descriptions of this link often treat star formation as dependent on (among others) local conditions, such as the gas column density (Skillman 1987) or the cooling time (Schaye 2001), or assume a more global dependency on the galaxy dynamics (e.g., Toomre- $Q$ or shear; Kennicutt 1989; Hunter et al. 1998). Our high-resolution data will allow a direct comparison with maps of the (resolved) stellar distribution of young stars.

In Section 2 we describe the observations, data reduction and data products. Section 3 compares the data with previous observations and highlights the new aspects of this data set. We also compare our data with the Chynoweth et al. (2008) deep GBT observations, and discuss a number of low-mass $\mathrm{HI}$ clouds visible in these data sets. In Section 4 we compare the H I column densities with stellar density maps and relate the profile velocity width in our data with those found in DLAs. Finally, we summarize our results in Section 5.

\section{Observations, Data Reduction, and Presentation}

The M81 triplet and its immediate surroundings were observed as part of a large 105-pointing mosaic covering $3^{\circ} \times 3^{\circ}(190 \times 190 \mathrm{kpc})$, centered on M81. This is the same area as observed by Chynoweth et al. (2008) using the GBT. The observations were done using the VLA in its C- and D-configurations between 2015 October and 2016 April.

The D-array observations took place in 10 separate observing sessions between 2015 October and December (project 15B-122); the C-configuration was used in 27 separate sessions during 2016 March and April (project 16A-073).

\subsection{Mosaic Layout and Observations}

We adopt a hexagonal Nyquist-sampled grid pattern of 105 separate pointings as shown in Figure 1. The horizontal and vertical distance between pointings is $13^{\prime}$ (half a primary beam width at $21 \mathrm{~cm}$ wavelength), with each row offset horizontally by half a spacing (one quarter primary beam width or $\sim 6.5$ ). The center positions of the pointings are listed in Table 1 and shown in Figure 1. The total observing area measures $\sim 3^{\circ} \times 3^{\circ}$, with the area inside the $50 \%$ sensitivity level measuring $\sim 2.7 \times 2.7$.

Each pointing was visited once per observing session. Pointings were observed in turn where in both R.A. and decl. directions every second row or column was skipped. This meant that to observe all pointings, the grid was covered a total of four times, each visit starting with a different pointing. This strategy ensures a more homogeneous $u v$-coverage and reduces the impact that any intermittant radio frequency interference (RFI) may have on any particular position.

Each pointing was visited for two minutes (including $\sim 10 \mathrm{~s}$ slewing and settling time). Every 15 pointings, including at the beginning and the end of each observing session, the complex gain/phase calibrator J0949+6614 was observed for one minute on-source. At the start of each session the primary flux and bandpass calibrator $3 \mathrm{C} 147$ was observed for two minutes 


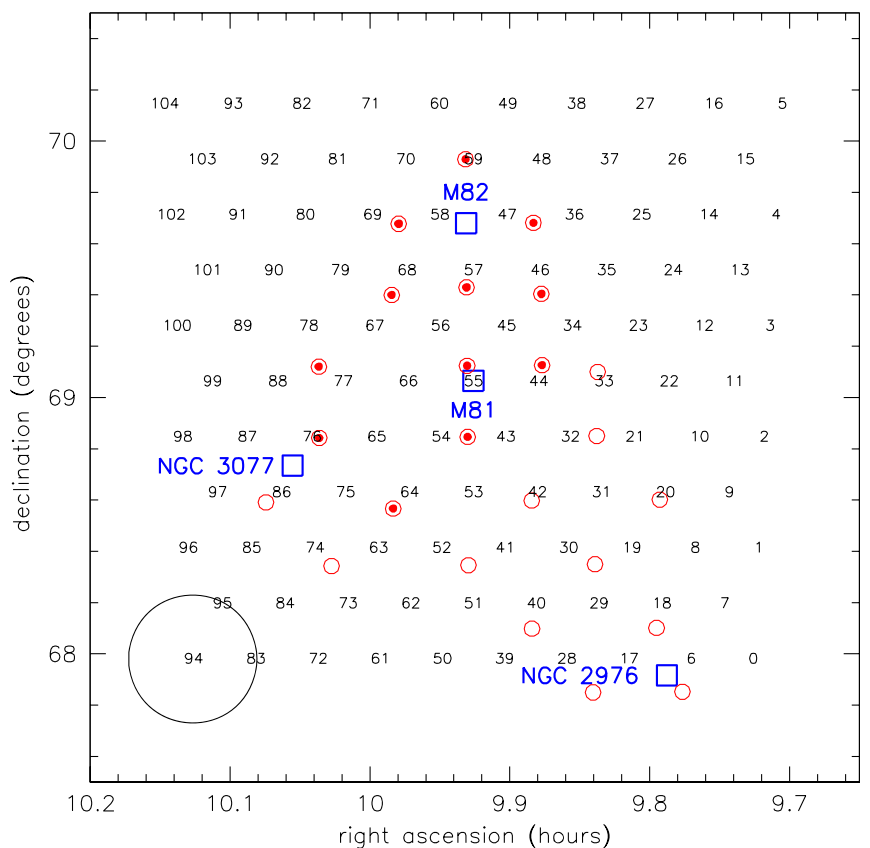

Figure 1. Mosaic pointings. Numbers indicate the central positions of the pointings listed in Table 1. Red dotted circles indicate the positions of the mosaic presented in Yun et al. (1994). Red open circles show the additional mosaic pointings described in Yun et al. (2000) and also presented in Chynoweth et al. (2008). The large circle in the lower left shows the size of the primary beam of a single pointing. The positions of M81, M82, NGC 3077, and NGC 2976 are indicated by blue squares.

on-source. The total duration of each observing session was $4 \mathrm{hr}$.

Combining all observing sessions, this resulted in a total integration time of 20 min per pointing for the D-array and 54 min for the $\mathrm{C}$-array, with a total integration time for the entire mosaic of $40 \mathrm{hr}$ for the D-array and $108 \mathrm{hr}$ for the C-array.

The WIDAR correlator was used in combination with the L-band receivers. We used the 8-bit correlator setup. An 8192channel, dual polarization subband was used to observe the $\mathrm{HI}$ line at $0.4 \mathrm{~km} \mathrm{~s}^{-1}(1.953 \mathrm{kHz})$ channel width over a $3200 \mathrm{~km} \mathrm{~s}^{-1}(16 \mathrm{MHz})$ bandwidth. In addition, eight spectral windows were allocated to observe the full polarization continuum at $1 \mathrm{MHz}$ resolution. A $4 \mathrm{MHz}$ subband was used to observe the $\mathrm{OH}$ and radio recombination lines. In this paper we discuss the $\mathrm{H}$ I observations only.

\subsection{Calibration and Flagging}

We extracted the HI data from each observing session's measurement set and averaged the $u v$ samples to an integration time of $10 \mathrm{~s}$. We ran the standard scripted VLA calibration pipeline (version 1.3.8) using CASA (McMullin et al. 2007) version 4.6.0. The pipeline was modified slightly to interpolate over $\sim 30 \mathrm{~km} \mathrm{~s}^{-1}$ of Galactic absorption in the 3C147 observations. The standard flagging set-up in the pipeline removed some of the bright H I target emission, so at this stage only calibrator pointings were automatically flagged.

The data were Hanning-smoothed prior to calibration. After calibration, every second channel was discarded, resulting in 4096 independent channels with a velocity spacing and resolution of $0.8 \mathrm{~km} \mathrm{~s}^{-1}$.
Due to issues with the CASA mosaicking routines, we exported all calibrated data to the Miriad (Sault et al. 1995) reduction package and used this for the rest of our data reduction. The conversion to Miriad format corrected all velocities from topocentric to barycentric, with the full velocity range of the $\mathrm{HI}$ data sets going from $-1735 \mathrm{~km} \mathrm{~s}^{-1}$ to $+1645 \mathrm{~km} \mathrm{~s}^{-1}$ (radio definition of velocities). Self-calibration was used, which improved the ratio of peak flux to noise by a factor three.

We used the uvlin task to subtract the continuum using a second-order polynomial. We experimented with lower-order polynomials but found that these gave less satisfactory results for the bright and resolved emission of M82. To fit the continuum emission, we used two ranges spanning 1000 channels each, covering velocities from $-1325 \mathrm{~km} \mathrm{~s}^{-1}$ to $-415 \mathrm{~km} \mathrm{~s}^{-1}$ and from +490 to $+1315 \mathrm{~km} \mathrm{~s}^{-1}$, respectively. This is well away from the velocity range where $\mathrm{HI}$ is expected: the deep GBT observations by Chynoweth et al. (2008) detect H I in the velocity range between -250 and $+340 \mathrm{~km} \mathrm{~s}^{-1}$. Later inspection of our data validated our choice.

As the target data were not flagged during the calibration stage, some flagging was done at this stage using the continuumsubtracted data. We used the task pgflag with its default settings to do a conservative SumThreshold flagging (Offringa et al. 2010) at $7 \sigma$, followed by flagging of visibilities with fewer than three unflagged neighbor visibilities. We then flagged time intervals or channels with less than $20 \%$ good data. We checked that no H I line visibilities were flagged. Inspection of the data showed that this procedure removed most of the artefacts from the $u v$-data, but some additional flagging was necessary to remove a number of more localized artefacts. Specifically, we flagged all visibilities with an amplitude $>8 \mathrm{Jy}$, all visibilities from baseline ea03-ea24, and the LL polarization of baseline ea18-ea24 below a $u v$-distance of $2 \mathrm{k} \lambda$.

A remaining subtle large-scale spatial ripple over the entire mosaic could not be readily identified in the $u v$-data. We therefore created an average $u v$-data set by averaging spectral channels 1000-1500 and produced a single-channel image. Inspection of the Fourier transform of this image showed the ripple to originate in high-amplitude visibilities between $200 \lambda$ and $400 \lambda$. The spatial scales with which these correspond make it conceivable that the ripples are due to some residual solar interference. We therefore flagged in the averaged $u v$ data set all visibilities in this range with amplitudes $>0.4 \mathrm{Jy}$. This flagged, averaged data set was used as a mask to flag the corresponding visibilities in the full data set. The resulting $u v$ data set was used to produce image cubes.

\subsection{Deconvolution and Clean Masks}

The invert task was used to produce the dirty beam and data cubes of the mosaic, combining all pointings of the C- and D-array. We adopted a pixel size of $5^{\prime \prime}$ and a channel width of $2 \mathrm{~km} \mathrm{~s}^{-1}$ over the velocity range $-400 \mathrm{~km} \mathrm{~s}^{-1}$ to $+450 \mathrm{~km} \mathrm{~s}^{-1}$. This resulted in a data cube of $2406 \times 2370 \times 425$ pixels. We produced cubes using natural weighting (robust $=2.0$ in the Miriad definition) and a higher-resolution version using robust $=0.5$. For convenience, we refer to these as the "natural-weighted" and the "robust-weighted" cubes, respectively.

We used the mossdi task to deconvolve the mosaicked cube. The aim was to clean the data cube deeply, to avoid residual-scaling effects (see Jörsäter \& van Moorsel 1995; Walter et al. 2008). Due to the large area involved, the desire to not clean large amounts of noise, and to minimize the possibility of clean-bias, we created masks to indicate areas 
Table 1

Pointing Center Coordinates

\begin{tabular}{|c|c|c|c|c|c|}
\hline Pointing & $\alpha \underset{\mathrm{h} \mathrm{m} \mathrm{s}}{(2000.0)}$ & $\begin{array}{c}\delta(2000.0) \\
0,11\end{array}$ & Pointing & $\alpha \underset{\mathrm{h} \mathrm{m} \mathrm{s}}{2000.0)}$ & $\begin{array}{c}\delta(2000.0) \\
0,11\end{array}$ \\
\hline 0 & $0943 \quad 32.98$ & +675857.88 & 52 & 095654.75 & +682456.73 \\
\hline 1 & $09 \quad 43 \quad 19.24$ & +682456.73 & 53 & 095533.20 & +683756.15 \\
\hline 2 & 094304.92 & +685055.57 & 54 & 095656.34 & +685055.57 \\
\hline 3 & 094249.99 & +69 1654.42 & 55 & 095533.20 & +690354.99 \\
\hline 4 & 094234.41 & +694253.26 & 56 & 095658.00 & +691654.42 \\
\hline 5 & $0942 \quad 18.13$ & +700852.11 & 57 & 095533.20 & +692953.84 \\
\hline 6 & 094613.02 & +675857.88 & 58 & 095659.73 & +694253.26 \\
\hline 7 & 094446.96 & +681157.30 & 59 & 095533.20 & +6955 52.69 \\
\hline 8 & 094602.34 & +682456.73 & 60 & 095701.54 & +700852.11 \\
\hline 9 & 094434.49 & +683756.15 & 61 & 095933.27 & +675857.88 \\
\hline 10 & 094551.21 & +685055.57 & 62 & 095814.76 & +681157.30 \\
\hline 11 & 094421.50 & +690354.99 & 63 & 095937.85 & +682456.73 \\
\hline 12 & 094539.59 & +691654.42 & 64 & 095817.87 & +683756.15 \\
\hline 13 & 094407.94 & +692953.84 & 65 & 095942.62 & +685055.57 \\
\hline 14 & 094527.47 & +694253.26 & 66 & 095821.12 & +690354.99 \\
\hline 15 & 094353.79 & +695552.69 & 67 & 095947.60 & +691654.42 \\
\hline 16 & 094514.81 & +700852.11 & 68 & 095824.51 & +692953.84 \\
\hline 17 & 094853.07 & +675857.88 & 69 & 095952.79 & +694253.26 \\
\hline 18 & 094728.52 & +681157.30 & 70 & 095828.05 & +695552.69 \\
\hline 19 & 094845.44 & +682456.73 & 71 & 095958.22 & +700852.11 \\
\hline 20 & $0947 \quad 19.17$ & +683756.15 & 72 & 100213.32 & +675857.88 \\
\hline 21 & 094837.49 & +685055.57 & 73 & 100056.31 & +681157.30 \\
\hline 22 & 094709.42 & +690354.99 & 74 & 100220.95 & +682456.73 \\
\hline 23 & 094829.19 & +691654.42 & 75 & 100102.55 & +683756.15 \\
\hline 24 & 094659.26 & +692953.84 & 76 & 100228.90 & +685055.57 \\
\hline 25 & 094820.54 & +694253.26 & 77 & 100109.04 & +690354.99 \\
\hline 26 & 094648.64 & $\begin{array}{r}+695552.69 \\
\end{array}$ & 78 & 100237.20 & +691654.42 \\
\hline 27 & 094811.49 & +700852.11 & 79 & 100115.82 & +692953.84 \\
\hline 28 & 095133.12 & +675857.88 & 80 & 100245.85 & +694253.26 \\
\hline 29 & $\begin{array}{lll}09 & 50 & 10.08\end{array}$ & +681157.30 & 81 & 100122.90 & +695552.69 \\
\hline 30 & 095128.54 & +682456.73 & 82 & 100254.90 & +700852.11 \\
\hline 31 & 095003.84 & +683756.15 & 83 & 100453.37 & +675857.88 \\
\hline 32 & 095123.77 & +685055.57 & 84 & 100337.87 & +681157.30 \\
\hline 33 & 094957.35 & +690354.99 & 85 & 100504.05 & +682456.73 \\
\hline 34 & $0951 \quad 18.79$ & +691654.42 & 86 & 100347.22 & +683756.15 \\
\hline 35 & 094950.57 & +692953.84 & 87 & 100515.19 & +685055.57 \\
\hline 36 & 095113.60 & +694253.26 & 88 & 100356.97 & +690354.99 \\
\hline 37 & 094943.49 & +695552.69 & 89 & 100526.80 & +691654.42 \\
\hline 38 & 095108.17 & +700852.11 & 90 & 100407.14 & +692953.84 \\
\hline 39 & 095413.17 & +675857.88 & 91 & 100538.92 & +694253.26 \\
\hline 40 & 095251.64 & +681157.30 & 92 & 100417.75 & +695552.69 \\
\hline 41 & 095411.64 & +682456.73 & 93 & 100551.58 & +700852.11 \\
\hline 42 & 095248.52 & +683756.15 & 94 & 100733.41 & +675857.88 \\
\hline 43 & 095410.05 & +685055.57 & 95 & 100619.43 & +681157.30 \\
\hline 44 & 095245.27 & +690354.99 & 96 & 100747.15 & +682456.73 \\
\hline 45 & 095408.39 & +691654.42 & 97 & 100631.90 & +683756.15 \\
\hline 46 & 095241.88 & +692953.84 & 98 & 100801.47 & +685055.57 \\
\hline 47 & 095406.66 & +694253.26 & 99 & 100644.89 & +690354.99 \\
\hline 48 & 095238.34 & +695552.69 & 100 & 100816.40 & +691654.42 \\
\hline 49 & 095404.85 & +700852.11 & 101 & 100658.45 & +692953.84 \\
\hline 50 & 095653.22 & $\begin{array}{r}+675857.88\end{array}$ & 102 & 100831.98 & $\begin{array}{r}+694253.26\end{array}$ \\
\hline \multirow[t]{2}{*}{51} & 095533.20 & +681157.30 & 103 & 100712.61 & +695552.69 \\
\hline & & & 104 & 100848.26 & +700852.11 \\
\hline
\end{tabular}

where deconvolution is allowed ("clean masks"). Due to the complex, extended and fragmented nature of the $\mathrm{H}$ I emission in the cube, we used a source-finding algorithm, specifically the smooth and clip algorithm implemented in the SoFiA software (Serra et al. 2015).

This applies a number of user-defined convolution combinations (Gaussian along the spatial axes and boxcar along the velocity axis) and, for each of these combinations, defines a binary mask by selecting all pixels above a user-defined threshold, expressed in multiples of the noise in the convolved data cube. The final mask is then the union of the masks belonging to each filter combination. Using a slight variation on the procedure described in Serra et al. (2012), we then applied a size filter to the SoFiA output mask by smoothing it 
spatially with the largest Gaussian beam used in the previous step. In the output mask, values $>0.5$ were then selected for the final mask.

This procedure creates masks objectively, but it is important that the final mask correctly isolates real signal. We have therefore extensively tested the algorithm described above on THINGS (The H I Nearby Galaxy Survey; Walter et al. 2008) data, where cubes, masks, and moment maps are readily available. THINGS is a multi-configuration VLA survey with a similar spatial and velocity resolution as our data set and should thus be representative. We used the source-finding algorithm and the THINGS data to create masked cubes and moment maps where the parameters were tweaked to most closely resemble the published THINGS results. We found that the optimum final mask is produced by a combination of masks using a $3 \sigma$ level at resolutions $(1,1,1),(1,1,2),(2,2,1)$ and $(2,2,2)$, where each triplet of numbers indicates the two spatial resolutions and the velocity resolution, in multiples of the original resolution.

To create the final clean masks, we cleaned the naturalweighted data cube down to $2 \sigma$ (without any pre-defined masks) and used the resulting cube as input for SoFiA. The task mossen was used to produce cubes showing the expected noise and gain (mosaic "primary beam" correction) values. We used the inverse of the noise cube as a weights cube in SoFiA to de-emphasize the higher noise values toward the edge of the mosaic and to prevent an excessive number of these noise peaks from entering the mask. The data set contains a number of channels with Galactic foreground HI. This foreground emission was included as part of the clean mask.

We then used mossdi again for a final deconvolution, using the clean mask, and this time cleaning down to $1 \sigma$ within the mask. The task restor was used to create the final, restored data cubes. For the robust-weighted data we used the same procedure and the same clean mask as for the natural-weighted data.

As noted, we used a deep cleaning limit to make residualscaling corrections (see Jörsäter \& van Moorsel 1995; Walter et al. 2008) negligible. Ianjamasimanana et al. (2017) have shown that (for THINGS data) cleaning to below $1.5 \sigma$ results in virtually identical fluxes from both residual-scaled and "standard," non-residual-scaled data. We checked this for our data by cleaning two representative channel maps to different depths of $1 \sigma, 0.75 \sigma$ and $0.5 \sigma$, creating a standard version as well as a residual-scaled version at each clean depth (using the residual scaling parameters given in Walter et al. 2008 and Ianjamasimanana et al. 2017).

We chose channel maps at $v=-92 \mathrm{kms}^{-1}$ and at $v=$ $+172 \mathrm{~km} \mathrm{~s}^{-1}$ which contain a significant amount of extended low-level emission. The first map is characteristic of the structures seen near M81, the second of those near M82. We determine the fluxes within the respective clean masks. For the M81 (M82) channel map we found that the residual-scaled fluxes derived using the three different depths agree to within $0.5(1.8) \%$. The three "standard" fluxes show a variation of $0.8(0.4) \%$. More importantly, we found that the $1 \sigma$ residual-scaled and standard fluxes agree to within $1.7(2.8) \%$, with the ratio decreasing to 0.4 $(0.6) \%$ for the $0.5 \sigma$ clean depth maps. In addition to the three depths just discussed, we checked the difference for a more shallow $2 \sigma$ limit, and found a difference of 6.2 (10.7)\% between standard and residual-scaled fluxes, consistent with the increasing relevance of residual-scaling for shallow clean limits. We therefore concluded that the standard fluxes derived here using a $1 \sigma$ clean were virtually identical to the residual-scaled fluxes, in agreement with Ianjamasimanana et al. (2017), and could be used in our further analysis.

\subsection{Beam Size and Sensitivity}

The natural-weighted $\mathrm{C}+\mathrm{D}$ data give a synthesized beam size of 38 "! $1 \times 30$ ". 9 , and a beam position angle (PA) of $75^{\circ} .5$. Assuming a distance of $3.63 \mathrm{Mpc}$ (Karachentsev et al. 2004), this corresponds to a linear resolution of $0.67 \times 0.54 \mathrm{kpc}$. The beam size for the robust-weighted data is 24 ". $3 \times 20$ ". 0 with a beam PA of $80^{\circ} .7$. The corresponding linear resolution is $0.43 \times 0.35 \mathrm{kpc}$. The noise in a $2 \mathrm{~km} \mathrm{~s}^{-1}$ channel is $1.17 \mathrm{mJy} \mathrm{beam}^{-1}$ for the natural-weighted cube and $1.25 \mathrm{mJy} \mathrm{beam}^{-1}$ for the robust data. These values are close to the theoretical noise. The corresponding column density sensitivities are $2.2 \times 10^{18} \mathrm{~cm}^{-2}$ (natural) and $5.7 \times 10^{18} \mathrm{~cm}^{-2}$ (robust). These are $1 \sigma$ values over a single $2 \mathrm{~km} \mathrm{~s}^{-1}$ channel. More representative sensitivies are given by $3 \sigma$ and $16 \mathrm{~km} \mathrm{~s}^{-1}$ (8 unaveraged channels) limits. These are $5.3 \times 10^{19} \mathrm{~cm}^{-2}$ (natural) and $1.4 \times 10^{20} \mathrm{~cm}^{-2}$ (robust). For unresolved sources, these noise levels imply a $5 \sigma \mathrm{HI}$ mass limit of $3.4 \cdot 10^{3}\left(W /\left[10 \mathrm{~km} \mathrm{~s}^{-1}\right]\right) M_{\odot}$ for the natural weighting, and a limit of $8.9 \times 10^{3}\left(W /\left[10 \mathrm{~km} \mathrm{~s}^{-1}\right]\right) M_{\odot}$ for the robust weighting. Here $W$ is the width of the $\mathrm{HI}$ profile in $\mathrm{km} \mathrm{s}^{-1}$.

Selected channel maps of the natural-weighted cube are shown in Figure 2 to give an overview of the M81 triplet data set. The channel maps clearly show the regular rotation of the inner parts of M81, the streaming motions in the outer arms and the connection with NGC 3077 in the southern part. This is in great contrast with the more chaotic and extended distribution of the $\mathrm{HI}$ in the northern part, including the connection with M82. The diffuse gas around M82 is visible over a large range in velocity. We can also clearly see the presence of Galactic foreground emission in a number of channels around velocities of $\sim-60$ and $\sim 0 \mathrm{~km} \mathrm{~s}^{-1}$.

\subsection{Moment Maps and Galactic Foreground}

To create moment maps we use a modified version of the clean mask with the Galactic foreground emission removed. Figure 2 shows that this emission is present at two distinct velocities. The main component is at $\sim 0 \mathrm{~km} \mathrm{~s}^{-1}$, with a second, fainter component at $\sim-58 \mathrm{~km} \mathrm{~s}^{-1}$. Specifically, from -64 to $-60 \mathrm{~km} \mathrm{~s}^{-1}$, Galactic emission is present in one corner of the image, without overlapping the M81 triplet area. We masked the Galactic signal manually for these channels. From -58 to $-48 \mathrm{~km} \mathrm{~s}^{-1}$, and again from -10 to $+8 \mathrm{~km} \mathrm{~s}^{-1}$, the Galactic emission is bright and overlaps the M81 triplet area. These channels were masked completely. The channels in between these components, from -46 to $-12 \mathrm{~km} \mathrm{~s}^{-1}$, were not affected. We did not attempt to interpolate the emission in the blanked channel maps due to the relatively small velocity range that was affected, and the complexity of the structures in these maps. In the rest of the cube no Galactic contamination is present and the remaining channels were not affected.

We used the updated mask to create the moment maps of both the natural-weighted and robust-weighted data using the moment task in Miriad. For both weightings we create zeroth (total intensity), first (intensity-weighted mean velocity), and second (velocity dispersion) moment maps. We used the gain 
(a)

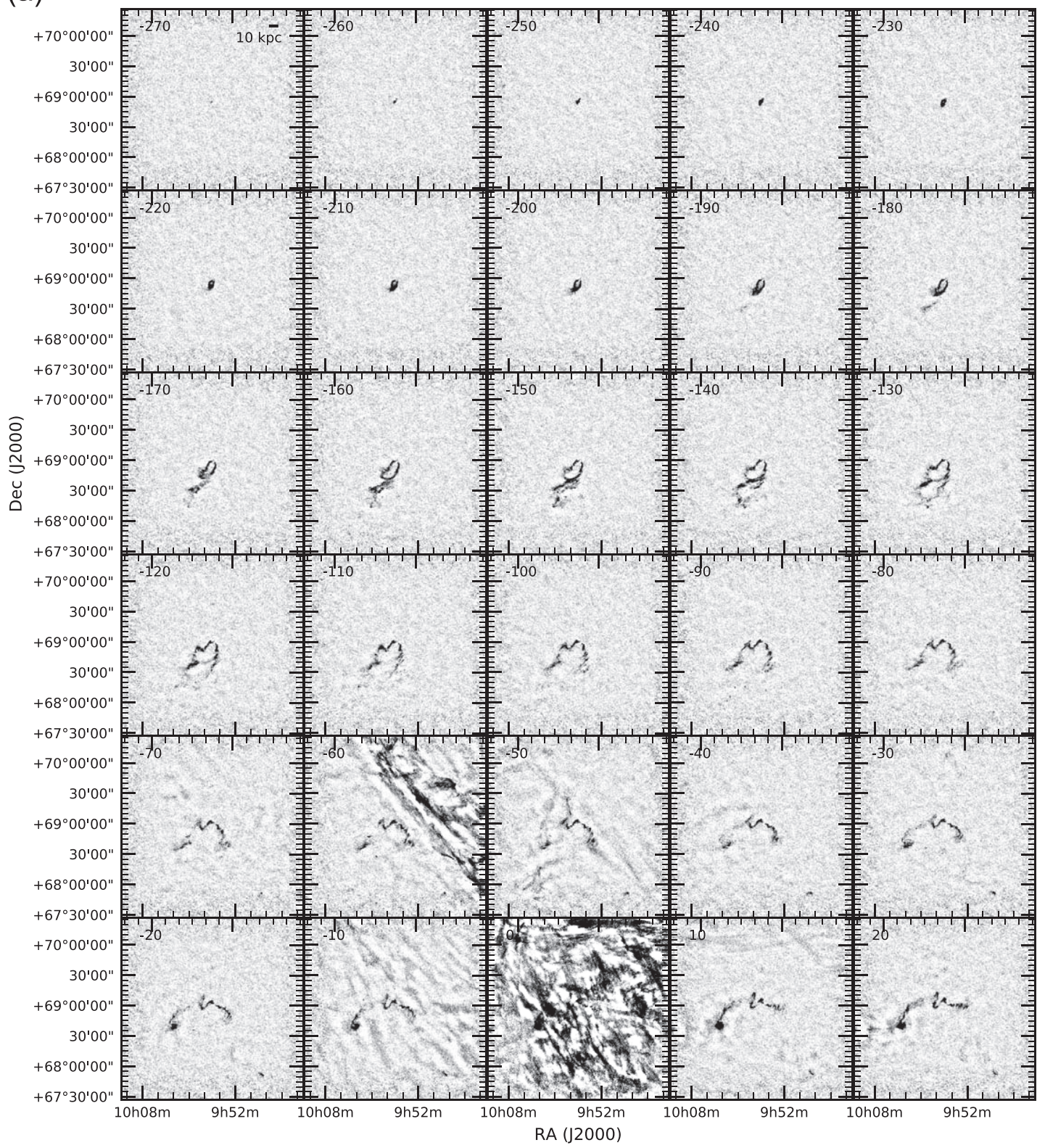

Figure 2. (a) Selected channel maps from the natural-weighted data. Every fifth channel is shown. The grayscale runs from $-0.5 \mathrm{mJy}$ beam ${ }^{-1}$ (white) to $+7 \mathrm{mJy}_{\text {beam }}^{-1}$ (black). The velocity of the channel in $\mathrm{km} \mathrm{s}^{-1}$ is shown in the top-left corner of each sub-panel. Only the full-sensitivity area of the mosaic is shown. The scale-bar in the top-left panel indicates $10 \mathrm{kpc}$. The emission of M82 extends to about $350 \mathrm{~km} \mathrm{~s}^{-1}$ (not shown here).

cube produced by mossen to mask spurious noise peaks toward the edges of the mosaic. We only retained signal that was present in more than three channels at each spatial position. In addition, we created total intensity maps using the original clean masks (i.e., with the Galactic emission still included). These could be used to gauge the effect of blanking the Galactic emission channels.

Due to different numbers of channels contributing to each of the pixels in the moment maps, the noise in an integrated intensity (zeroth moment) map is not constant. We derive the noise as follows. For a zeroth moment map based on independent channels (as is the case here), the noise in a pixel $\sigma_{\text {momo }}$ is defined as $\sigma_{\text {mom } 0}=\sigma_{\text {chan }} \cdot \sqrt{N_{\text {chan }}}$, where $\sigma_{\text {chan }}$ is the noise in a single channel and $N_{\text {chan }}$ the number of channels contributing to a moment-map pixel. (A zeroth moment is a sum, not an average, which is why the noise increases.) The signal-to-noise ratio $\mathrm{S} / \mathrm{N}$ of each pixel can be derived by dividing the zeroth-moment map by a map of $\sigma_{\text {chan }}$ (taking care to treat the units consistently, i.e., the $\sigma_{\text {chan }}$ map should also have Jy beam ${ }^{-1} \mathrm{~km} \mathrm{~s}^{-1}$ units, to avoid introducing extra factors equal to the channel separation). We then select all pixels in the $\mathrm{S} / \mathrm{N}$ map with values between 2.5 and 3.5. We take the mean value of the corresponding pixels in the zeroth-moment map to represent the average $\mathrm{S} / \mathrm{N}=3$ column density sensitivity. We find a value of $15.0 \pm 5.5 \mathrm{mJy}$ beam $\mathrm{km} \mathrm{s}^{-1}$, corresponding to a column density sensitivity of $1.42 \cdot 10^{19} \mathrm{~cm}^{-2}$ for the natural-weighted data. A similar 
(b)

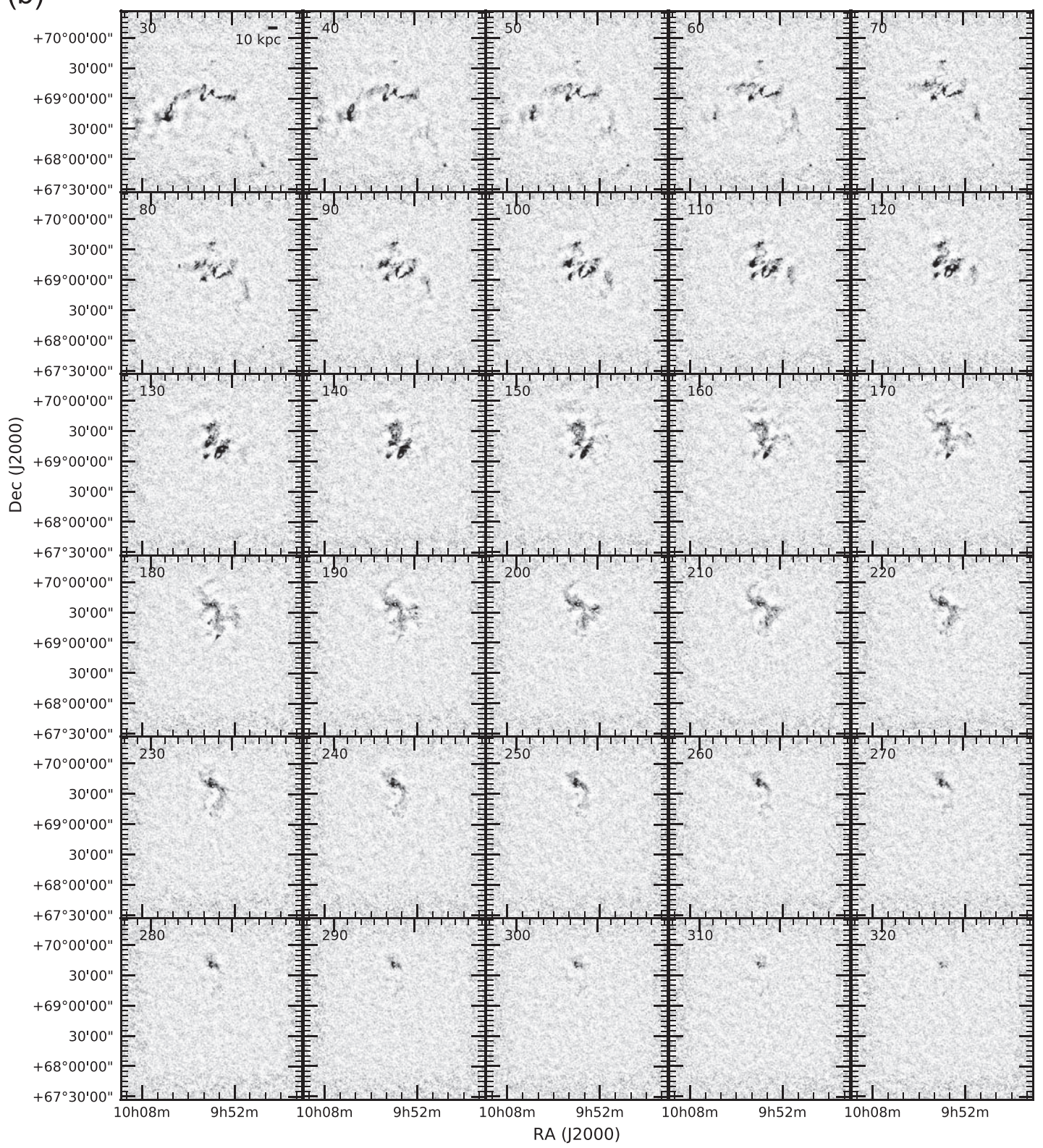

Figure 2. (Continued.)

procedure for the robust-weighted data gives a $\mathrm{S} / \mathrm{N}=3$ value of $24.2 \pm 6.1 \mathrm{mJy}$ beam $\mathrm{km} \mathrm{s}^{-1}$, corresponding to a column density of $5.54 \cdot 10^{19} \mathrm{~cm}^{-2}$.

To ensure a homogeneous column density limit across the maps, we apply a zeroth-moment value cutoff of $1.5 \times 10^{19} \mathrm{~cm}^{-2}$ to the natural-weighted maps and $5.5 \times 10^{19} \mathrm{~cm}^{-2}$ to the robust map. The resulting maps are also applied as masks to the respective first- and second-moment maps.

The natural-weighted moment maps are presented in Figures 3 (left) (zeroth-moment map), 4 (first-moment map) and 5 (secondmoment map). These maps are further discussed in Section 3.1. In Figure 6 we show a false-color representation of the robustweighted zeroth-moment map overlaid on an SDSS optical image. A summary of the optical positions and sizes is given in Table 2 .

In Figure 7, we compare the two zeroth-moment maps created using the masks with and without the Galactic foreground emission. Comparison of the two moment maps shows the effect of the Galactic masking. For example, the appearance of NGC 2976 seemingly having two separate H I components is due to the masking used, and similarly, some emission is missing along the minor axis of M81. The final effect of this masking on properties like total HI masses is, however, small compared to other uncertainties, as discussed in more detail in Section 3.3.

\subsection{D-array Cubes and Moment Maps}

We also produced more sensitive, lower-resolution versions of the cubes and maps using the D-array data and the shorter baselines from the $\mathrm{C}$-array data by selecting all data with a $u v$ distance $<5 \mathrm{k} \lambda$. For convenience, we refer to this data set as the "D-array" data.

We used the same procedure as for the $\mathrm{C}+\mathrm{D}$ data described above. The resulting natural-weighted deconvolved cube has a beam size of $94 \prime \prime 5 \times 76^{\prime \prime} 0$ with a beam PA of $78^{\circ} .2$. At the distance of M81, this corresponds to a resolution of 

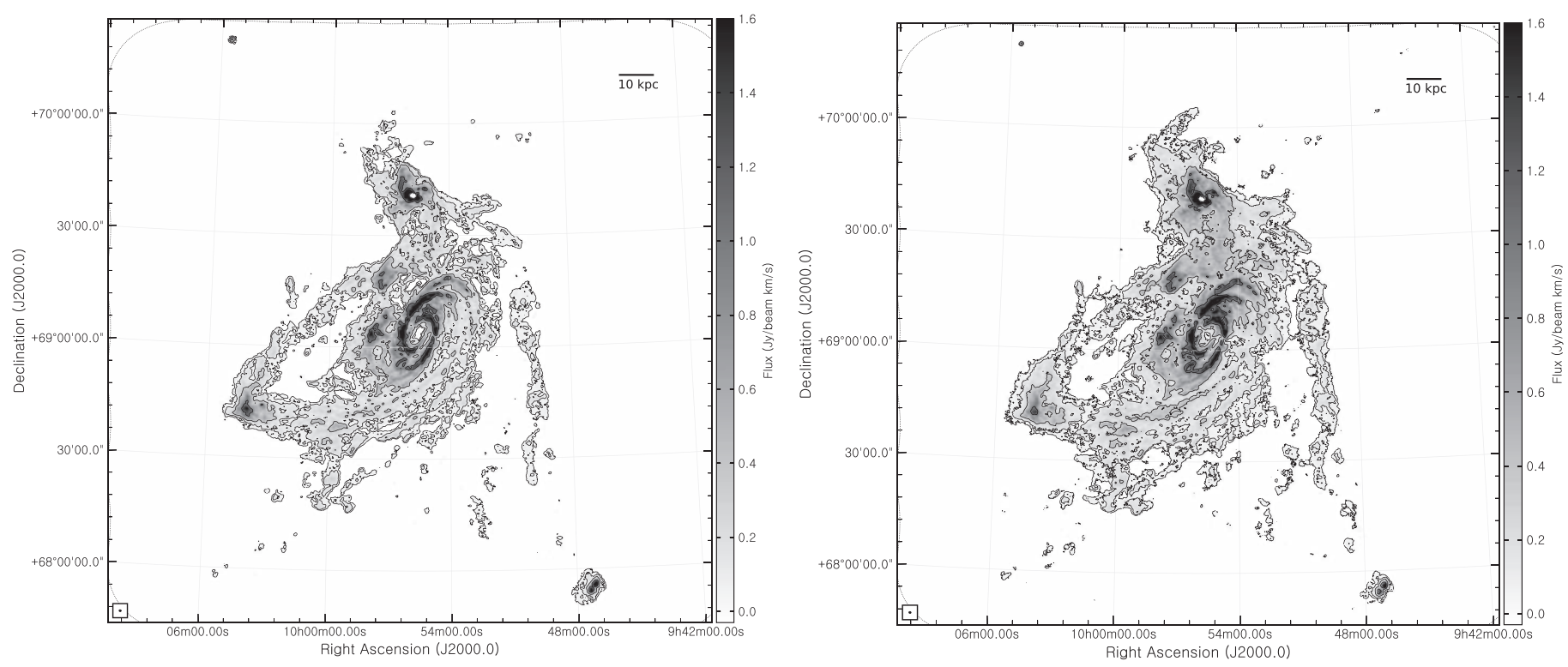

Figure 3. Left: natural-weighted integrated intensity (zeroth moment) map derived using the $\mathrm{C}+\mathrm{D}$ data. The grayscale runs from 0 (white) to 1.6 (black) Jy beam ${ }^{-1} \mathrm{~km} \mathrm{~s}^{-1}$. Contours levels are $0.0316 \times 10^{x} \mathrm{Jy} \mathrm{beam}^{-1} \mathrm{~km} \mathrm{~s}^{-1}$ where $x=(0,0.5,1,1.5)$. This corresponds to column densities of $(0.3,0.95,3,9.5) \times$ $10^{20} \mathrm{~cm}^{-2}$. Only the area inside the $50 \%$ sensitivity contour (dotted curve visible in the corners) is shown. Right: zero-spacing corrected zeroth moment map based on the natural-weighted C+D VLA and GBT data. Contours and grayscales as in left panel.

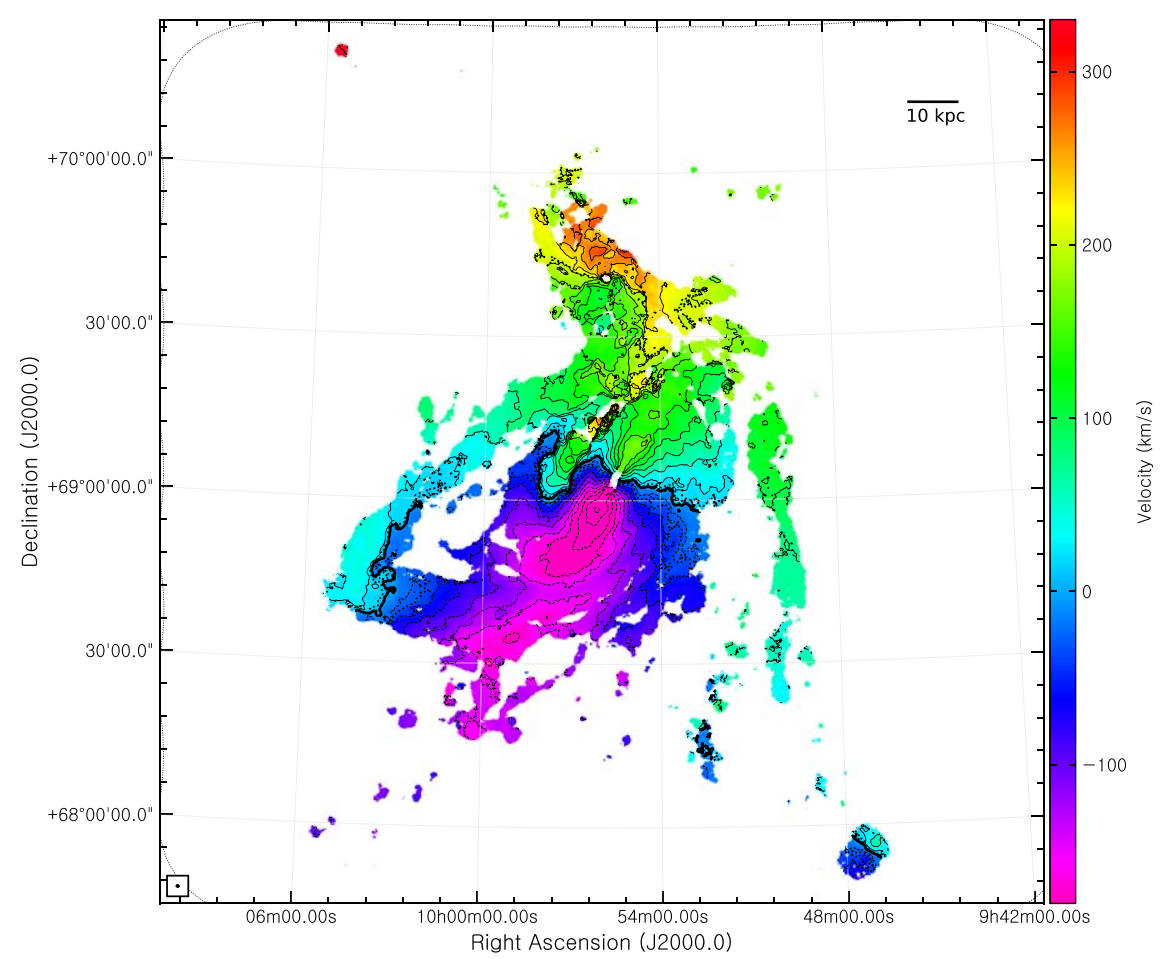

Figure 4. Natural-weighted intensity-weighted mean velocity (first moment) map derived using the $\mathrm{C}+\mathrm{D}$ data. The color scale runs from -180 to $330 \mathrm{~km} \mathrm{~s}{ }^{-1}$, as indicated by the color bar. Contour levels run from -250 to $+400 \mathrm{~km} \mathrm{~s}^{-1}$ and are spaced by $25 \mathrm{~km} \mathrm{~s}^{-1}$. Negative contours are dashed. The thick contour is at $0 \mathrm{~km} \mathrm{~s}^{-1}$. Only the area inside the $50 \%$ sensitivity contour (dotted curve visible in the corners) is shown.

$1.67 \times 1.34 \mathrm{kpc}$. For the D-array data we did not consider a robust weighting.

The noise in the natural-weighted data set is $1.81 \mathrm{mJy}_{\text {beam }}^{-1}$ for a single $2 \mathrm{~km} \mathrm{~s}^{-1}$ channel. This corresponds to a $1 \sigma, 1$ channel column density sensitivity of $5.6 \times 10^{17} \mathrm{~cm}^{-2}$, or a more representative $3 \sigma, 16 \mathrm{~km} \mathrm{~s}^{-1}$ (eight independent, unaveraged channels) limit of $1.3 \times 10^{19} \mathrm{~cm}^{-2}$.
For the moment maps calculation, we created new masks due to the increased prominence of the Galactic emission. From -66 to $-58 \mathrm{~km} \mathrm{~s}^{-1}$, Galactic emission covered part the field, without affecting the main M81 triplet emission, and this Galactic emission was masked by hand. The channels from -56 to -50 and from -8 to $+6 \mathrm{~km} \mathrm{~s}^{-1}$ were blanked completely. In between these ranges, from -48 to $-10 \mathrm{~km} \mathrm{~s}^{-1}$, no blanking was needed. 


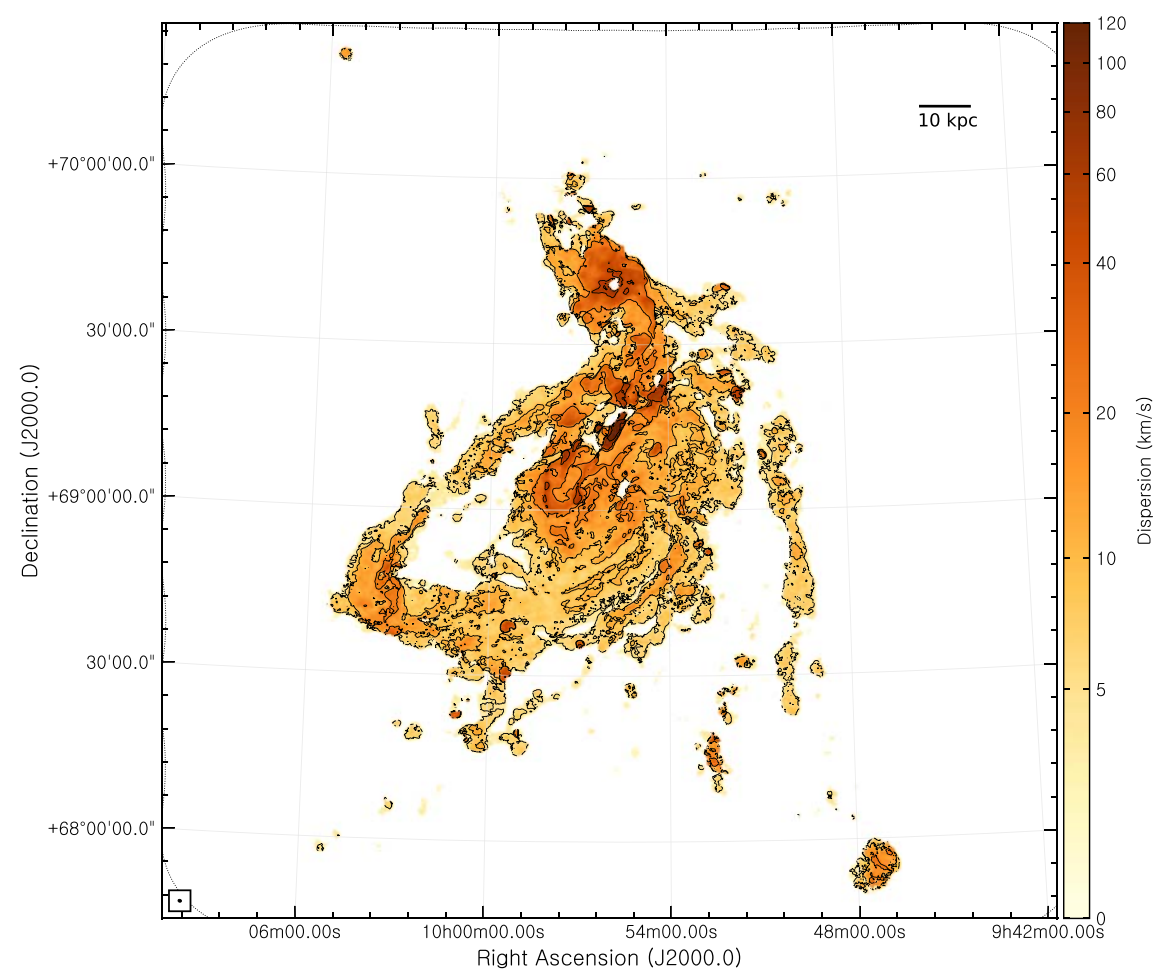

Figure 5. Natural-weighted velocity dispersion (second moment) map using the $\mathrm{C}+\mathrm{D}$ configurations. The color scale uses an arcsinh stretch, running from 0 (light) to 120 (dark) $\mathrm{km} \mathrm{s}^{-1}$. Contour levels are at $5,10,20,50$, and $100 \mathrm{~km} \mathrm{~s}^{-1}$. Only the area inside the $50 \%$ sensitivity contour (dotted curve visible in the corners) is shown.

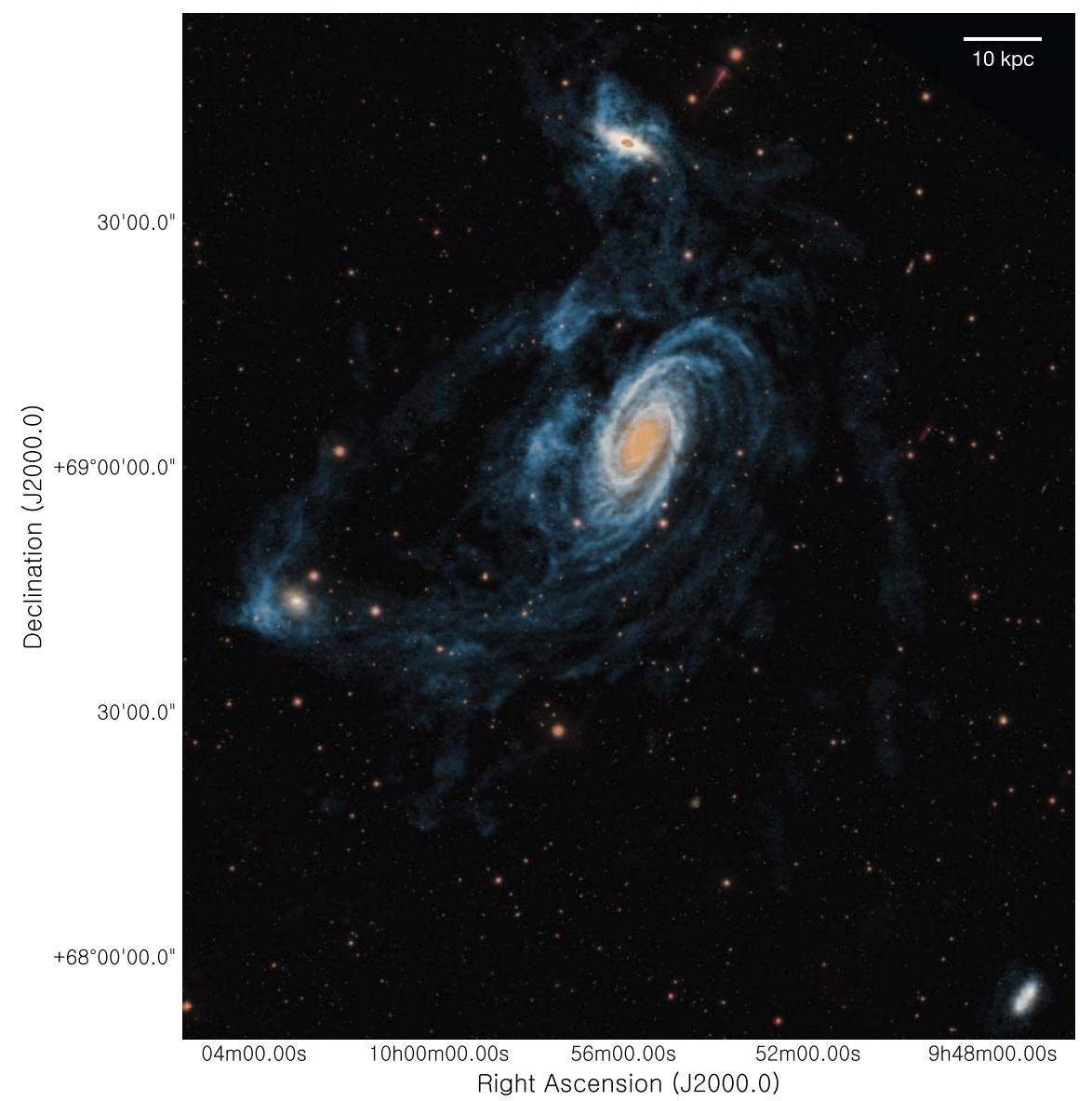

Figure 6. False-color overlay of the robust-weighted zeroth-moment map (in blue) on a color SDSS image of the M81 triplet. The area shown is slightly smaller than in Figure 3. 
Table 2

Properties of the Four Main Galaxies

\begin{tabular}{|c|c|c|c|c|c|c|}
\hline $\begin{array}{l}\text { Galaxy } \\
\text { (1) }\end{array}$ & $\begin{array}{c}\alpha(2000.0) \\
\left(\begin{array}{c}\mathrm{h} \mathrm{m} \mathrm{s} \\
)\end{array}\right. \\
(2)\end{array}$ & $\begin{array}{c}\delta(2000.0) \\
\left(\begin{array}{c}\circ \prime \prime \prime \\
(3)\end{array}\right)\end{array}$ & $\begin{array}{c}D_{\text {Holmberg }} \\
\left({ }^{\prime}\right) \\
(4)\end{array}$ & $\begin{array}{l}i_{\mathrm{opt}} \\
\left({ }^{\circ}\right) \\
(5)\end{array}$ & $\begin{array}{l}\mathrm{PA}_{\text {opt }} \\
\left({ }^{\circ}\right) \\
(6)\end{array}$ & $\begin{array}{c}V_{\text {sys }}^{\text {hel }} \\
\left(\mathrm{km} \mathrm{s}^{-1}\right) \\
(7)\end{array}$ \\
\hline M81 & 095533.2 & +690355 & $35.0 \times 14.4$ & 57 & 157 & -34 \\
\hline M82 & 095552.7 & +694046 & $13.4 \times 8.5$ & 82 & 65 & 203 \\
\hline NGC 3077 & 100319.1 & +684402 & $8.8 \times 8.0$ & 38 & 45 & 14 \\
\hline NGC 2976 & 094715.4 & +675459 & $9.7 \times 5.7$ & 61 & 143 & 3 \\
\hline
\end{tabular}

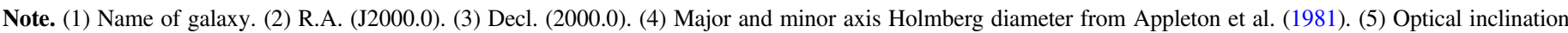
from Appleton et al. (1981).(6) Optical position angle from UGC (Nilson 1973). (7) Heliocentric systemic velocity from RC3 (de Vaucouleurs et al. 1991).

A small amount of manual blanking was needed for the channels at +8 and $+10 \mathrm{~km} \mathrm{~s}^{-1}$, where prominent Galactic emission covered a small part of the field. No further blanking was needed for the remaining channels, except for a small area immediately to the east of NGC 3077, where from +12 to $+34 \mathrm{~km} \mathrm{~s}^{-1}$ some aliasing and mosaicking artefacts were removed.

In the zeroth-moment map, the average value of $\mathrm{S} / \mathrm{N}=3$ pixels is $18.3 \pm 8.0 \mathrm{mJy}$ beam $\mathrm{km} \mathrm{s}^{-1}$, corresponding to an average column density of $2.83 \times 10^{18} \mathrm{~cm}^{-2}$. To achieve a homogeneous sensitivity, we blanked the zeroth-moment map, and the corresponding pixels in the first- and second-moment maps, at a column density value of $3.0 \times 10^{18} \mathrm{~cm}^{-2}$.

\subsection{Zero-spacing Corrections Using GBT Data}

Interferometers are limited in their ability to recover the total fluxes of objects, especially if these are extended compared to the size scale corresponding to the shortest baseline. Single-dish data are often used to correct the fluxes in the interferometric data and enhance extended structures. Here we use GBT data to apply this zero-spacing correction to our data.

As noted in Section 1, GBT observations of the survey area are published in Chynoweth et al. (2008). We could not, however, use the Chynoweth et al. (2008) data cube as an unflagged version (still including Galactic emission) was not available. For the zerospacing correction we therefore used the GBT data set covering the M81/M82 and NGC 2403 groups as published in Chynoweth et al. (2011) (though this data set incorporates the Chynoweth et al. 2008 data).

The channel spacing of the data set is $5.2 \mathrm{~km} \mathrm{~s}^{-1}$, with a

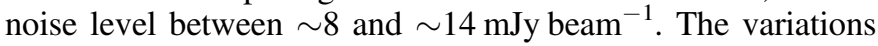
in noise level are due to the patching together of many different observations (see Figure 3 in Chynoweth et al. 2011). In the area covering the triplet, the data set is for all practical purposes equal to the Chynoweth et al. (2008) data, resulting in a noise level of $\sim 8 \mathrm{mJy}^{\text {beam }}{ }^{-1}$. For the GBT beam size of $9 ! 4$, this corresponds to a $1 \sigma, 1$ channel $\left(5.2 \mathrm{~km} \mathrm{~s}^{-1}\right)$ column density sensitivity of $2.5 \times 10^{17} \mathrm{~cm}^{-2}$.

We extracted the region corresponding to our VLA mosaic from this data set and regridded it to the spatial and spectral pixel size of the VLA mosaic. Note that this meant oversampling the GBT velocity channels by a factor $\sim 2.5$ to achieve a $2 \mathrm{~km} \mathrm{~s}^{-1}$ channel spacing. We combined the naturalweighted $\mathrm{C}+\mathrm{D}$ VLA data and the GBT data using the Miriad task immerge. This task combines the two image cubes in the Fourier plane, and optionally uses the range in spatial frequencies where the single-dish and interferometer data overlap to determine a scale factor to bring the single dish flux scale in agreement with the interferometer one. For our data we used a $u v$-range between 35 and $90 \mathrm{~m}$ for the overlap.
Comparing fluxes in the velocity range between -252 and $-102 \mathrm{~km} \mathrm{~s}^{-1}$ we found an optimal scale factor of 1.08 for the GBT data. Tests using different velocity ranges (excluding that of the Galactic emission) yielded similar values. The final, combined cube as produced by immerge had a noise level and resolution equal to that of the VLA $\mathrm{C}+\mathrm{D}$ data cube.

The increased prominence of Galactic foreground emission, and the presence of additional features introduced in the combined cube, meant we created a new mask to produce moment maps. As before, we used SoFiA, using the same settings, and applied the same size filter.

The velocity range from -450 to $-78 \mathrm{~km} \mathrm{~s}^{-1}$, and from +22 to $+450 \mathrm{~km} \mathrm{~s}^{-1}$ needed no additional blanking. Galactic emission dominated the velocity range from -62 to $-44 \mathrm{~km} \mathrm{~s}^{-1}$, and from -10 to $+20 \mathrm{~km} \mathrm{~s}^{-1}$. These channels were completely blanked. Finally, from -76 to -64 and from -42 to $-12 \mathrm{~km} \mathrm{~s}^{-1}$ Galactic emission was present but did not overlap with the triplet emission. Here the Galactic emission was identified and blanked by hand. In addition, a small aliasing effect toward the edge of the mosaic east of NGC 3077 was also removed by hand. Comparison with Section 2.5 shows that in the combined cube a substantially larger range in velocity is affected by Galactic emission.

This mask was then used to create moment maps, applying the same $\mathrm{S} / \mathrm{N}=3$ column density cut, and retaining only signal occurring over more than three consecutive channels.

The zeroth moment map is shown in Figure 3 (right panel). A comparison with the VLA-only map shown in the left panel of the same figure clearly shows that the artefact running along the minor axis of M81 due to the blanking of Galactic emission is more prominent in the zero-spacing corrected cube. Note that the increased blanking, along with the lower velocity resolution of the GBT data, affects the corrected first- and second-moment maps and in the rest of this paper we therefore only consider the VLA-only first- and second-moment maps as shown in Figures 4 and 5.

\subsection{Position-velocity Slices}

The moment maps presented here give a concise description of the morphology and kinematics of the H I in the M81 triplet. A disadvantage of these moment maps is that much information on the detailed velocity structure of the gas is lost. Moment maps along the two other (spatial) axes of the cube can show some of the global velocity structure of the emission but, due to the projection, detailed information on smaller-scale structures is lost here as well.

An alternative solution is to make use of position-velocity slices. These show the velocity structure of the gas along a spatial slice. In principle, these slices can be extracted from the data cube at any arbitrary position and position angle, fine-tuned to highlight 

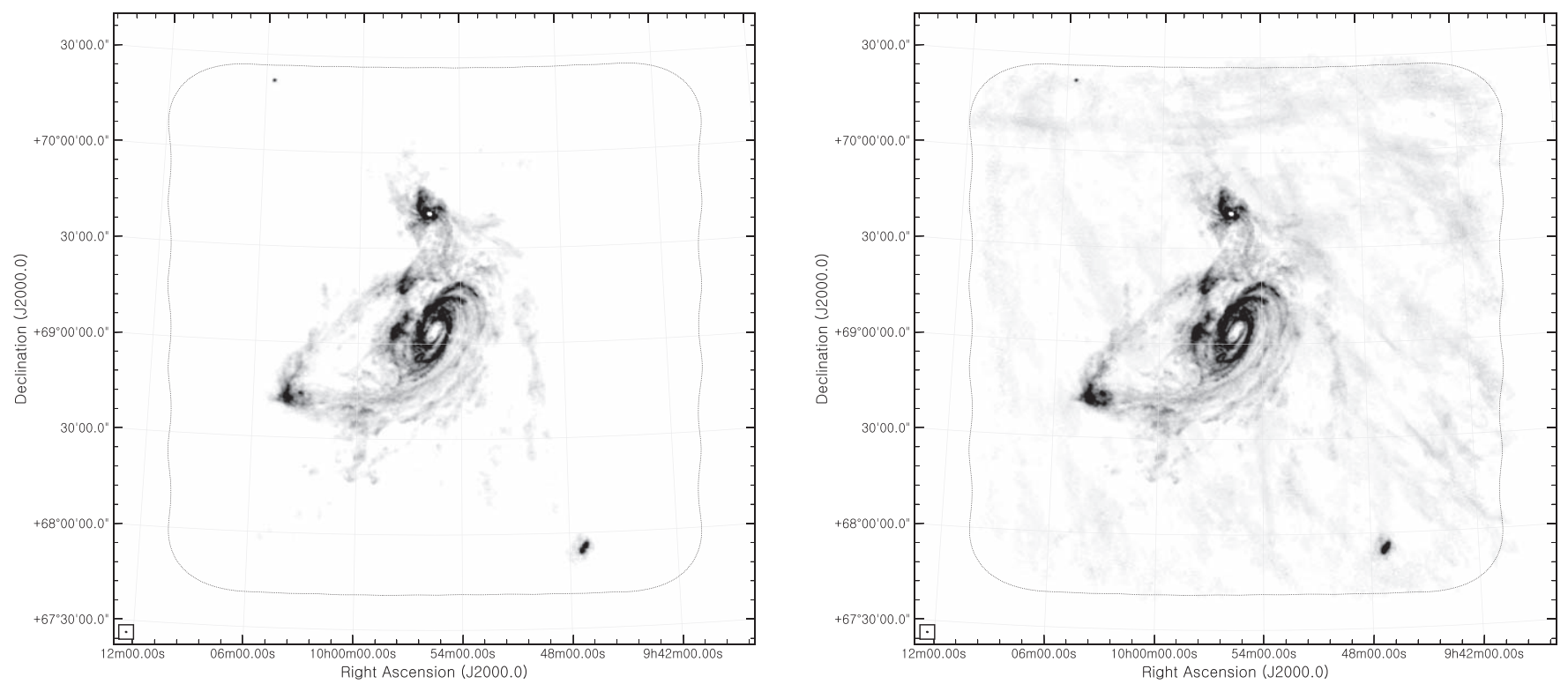

Figure 7. Comparison of $\mathrm{C}+\mathrm{D}$ natural-weighted zeroth-moment maps without (left panel) and with (right panel) the Galactic emission channels. In both panels, the grayscale runs from 0 (white) to 1.0 (black) Jy beam ${ }^{-1} \mathrm{~km} \mathrm{~s}^{-1}$. The beam is indicated in the lower-left corner. The dotted curve indicates the $50 \%$ sensitivity level of the mosaic area.

a particular feature. Here, we want to produce a general overview of the velocity structure of the triplet. Using the zero-spacing corrected natural-weighted $\mathrm{C}+\mathrm{D}$ data, we extracted a number of slices parallel to the major axis of M81, covering the full extent of the triplet along each slice. We assumed a major axis PA of $330^{\circ}$ (de Blok et al. 2008), which is also a reasonable approximation for the orientation on the sky of the entire triplet.

To keep the number of slices manageable and increase the signal-to-noise in each slice, we extracted slices with a perpendicular thickness of $140^{\prime \prime}$ (around four natural-weighted beams). We tested several slide thicknesses and found that the value of $140^{\prime \prime}$ gives a good compromise between increasing the signal-to-noise and preserving the visibility of small-scale features.

Most of the prominent velocity features in the triplet can be covered by 20 contiguous slices covering most of the eastern part of the triplet and a smaller fraction of the western part. Figure 8 shows the positions of the slices superimposed on the zeroth-moment map of the triplet. Slices are numbered from 1 to 20 , with slice 1 the easternmost slice, and slice number increasing to the west. Slice 15 is centered on the center of M81 and is located on the M81 major axis. Slices 6 and 7 pass close to the center of M82.

The slices' position-velocity diagrams are shown in Figure 9. The Galactic emission is clearly visible in all slices at velocities of $\sim 0$ and $\sim-50 \mathrm{~km} \mathrm{~s}^{-1}$. The leftmost part (negative velocites and negative offsets) of the panels corresponds to the southern part of the mosaic, the rightmost part (positive velocities and positive offsets) to the northern part. The increased noise in the very leftmost part of the slices is due to the decreased sensitivity at the southern edge of the mosaic. The rightmost (northern) edge is not shown due to a lack of features there. The position-velocity slices are discussed in more detail in Section 3.1.

\subsection{Additional Southeast Mosaic Pointings}

In addition to the main mosaic data, we also use additional data from project AW683 to extend the mosaic coverage further toward the southeast (SE). These data consist of a 16-pointing

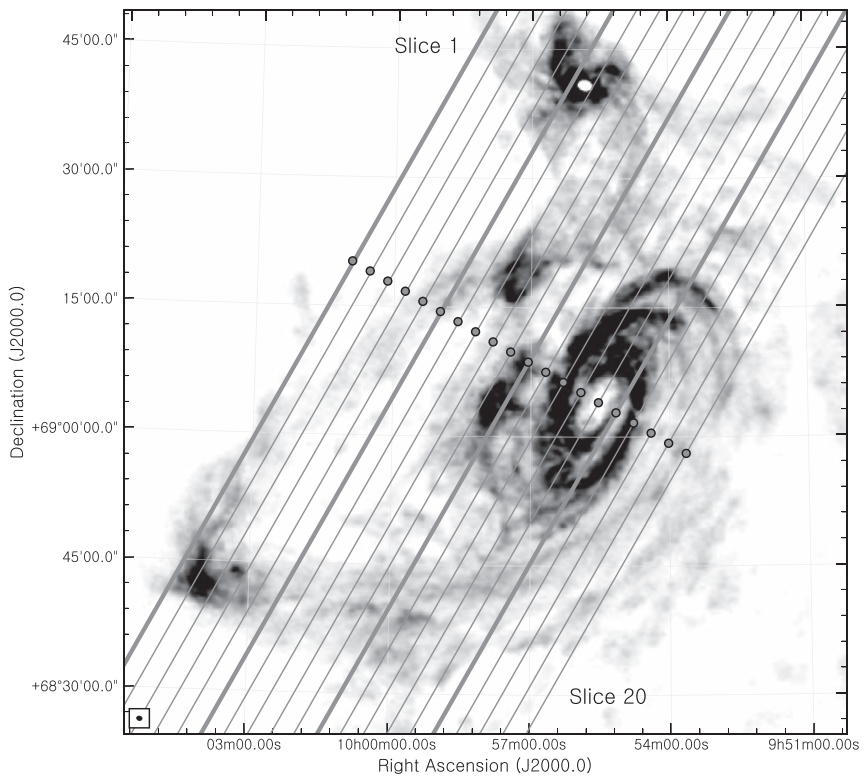

Figure 8. Central positions of the position-velocity slices presented in Figure 9, superimposed on a zeroth-moment map. The circles indicate the zeropoints for the offsets along the slices. For ease of reference, every fifth slice is shown using a thick line. Slice 1 is the easternmost slice, slice 20 the westernmost. Slice 15 is centered on the center of M81. Every slice is $140^{\prime \prime}$ thick and separated by the same amount from the adjacent slices. The lines shown here indicate the slices' centers. The position angle of the slices is $150^{\circ} 3$, corresponding to the position angle of the major axis of M81.

mosaic observed in C- and D-array and partly overlapping with the SE corner of the main mosaic (see Figure 10).

These data were taken in 2006 December (C-array) and 2007 April (D-array), when the VLA/EVLA transition was underway, meaning not all baselines were usable. The integration time was about 50 min per pointing in each of the two configurations. The observations were done with a channel spacing of $5 \mathrm{~km} \mathrm{~s}^{-1}$ between -355 and $+210 \mathrm{~km} \mathrm{~s}^{-1}$. The $\mathrm{C}$-array data did not significantly improve the signal-to-noise of 
(a)
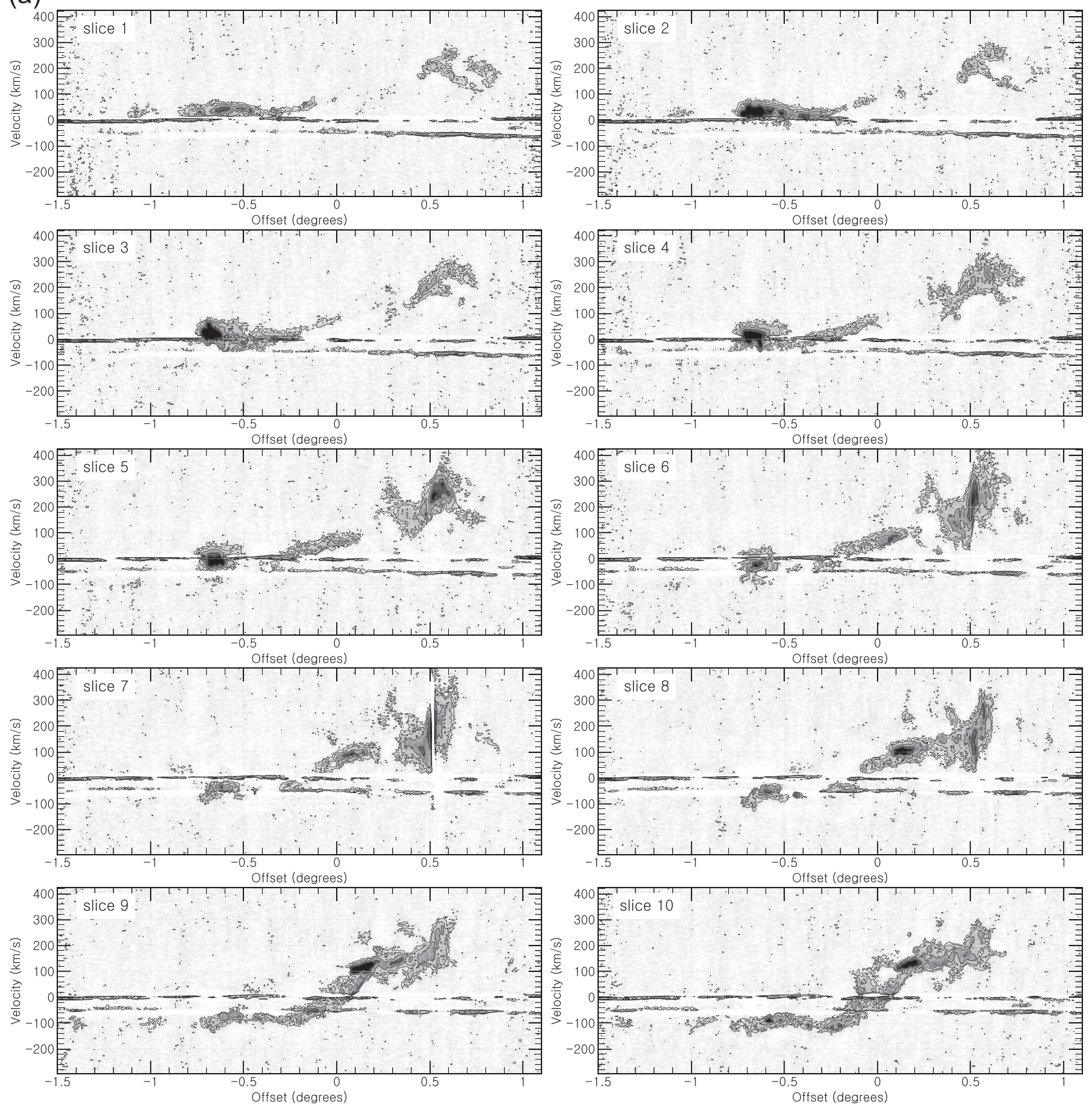

Figure 9. (a) Position-velocity slices covering part of the M81 triplet, as shown in Figure 8. Numbering of the slices is as shown in that figure. Negative offsets are toward the south, positive offsets to the north. The zero-point corresponds with the respective circles indicated in Figure 8 . The slices are $140 "$ thick, and emission is summed perpendicularly to each slice. The lowest contour shown is $0.015 \mathrm{Jy}_{\text {beam }}^{-1}$, corresponding to $3 \sigma$ in these summed slices. Contour levels then increase by factors of two. The grayscale runs from $-0.01 \mathrm{Jy} \mathrm{beam}^{-1}$ (white) to $+0.2 \mathrm{Jy} \mathrm{beam}^{-1}$ (black). Galactic emission is visible in all slices at 0 and $-50 \mathrm{~km} \mathrm{~s}^{-1}$. Increased noise in the leftmost part of the slices arises from decreased sensitivity due to the edge of the mosaic. (b) As in panel (a).

the final data set, so we do not consider these data any further. The central of the three easternmost pointings was severely affected by RFI, and we discard that pointing.

We subtracted a zeroth-order continuum fit, and produced a natural-weighted, D-array-only datacube using the remaining pointings and a channel spacing of $10 \mathrm{~km} \mathrm{~s}^{-1}$. The noise per $10 \mathrm{~km} \mathrm{~s}^{-1}$ channel is $1.1 \mathrm{mJy}$ beam $^{-1}$. We cleaned the cube down to $1.5 \sigma$ using mossdi in Miriad. The synthesized beam is $80^{\prime \prime} 2 \times 69^{\prime \prime} 2$, with a beam position angle of $30^{\circ} .0$. The column density limit of these data is $1.95 \times 10^{18} \mathrm{~cm}^{-2}(1 \sigma, 1$ channel of $10 \mathrm{~km} \mathrm{~s}^{-1}$ ), or, more representative, 1.17 . $10^{19} \mathrm{~cm}^{-2}\left(3 \sigma, 20 \mathrm{~km} \mathrm{~s}^{-1}\right.$ or 2 channels).

The integration time per pointing is approximately equal to those of the D-array observations of our mosaic; however, the noise level in the AW683 data is $~ 40 \%$ higher (taking into account the different channel widths used). This is due to a 
(b)
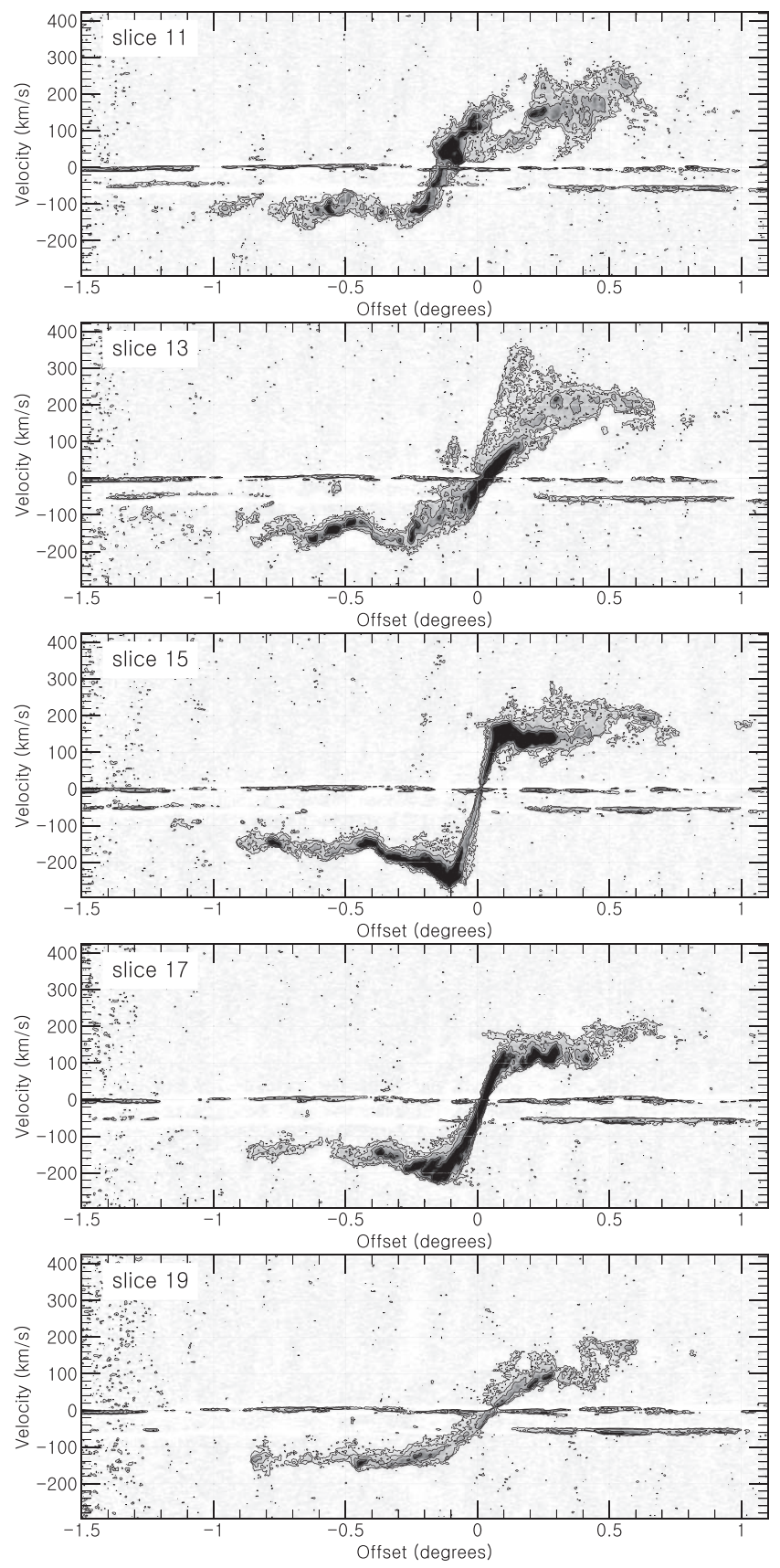
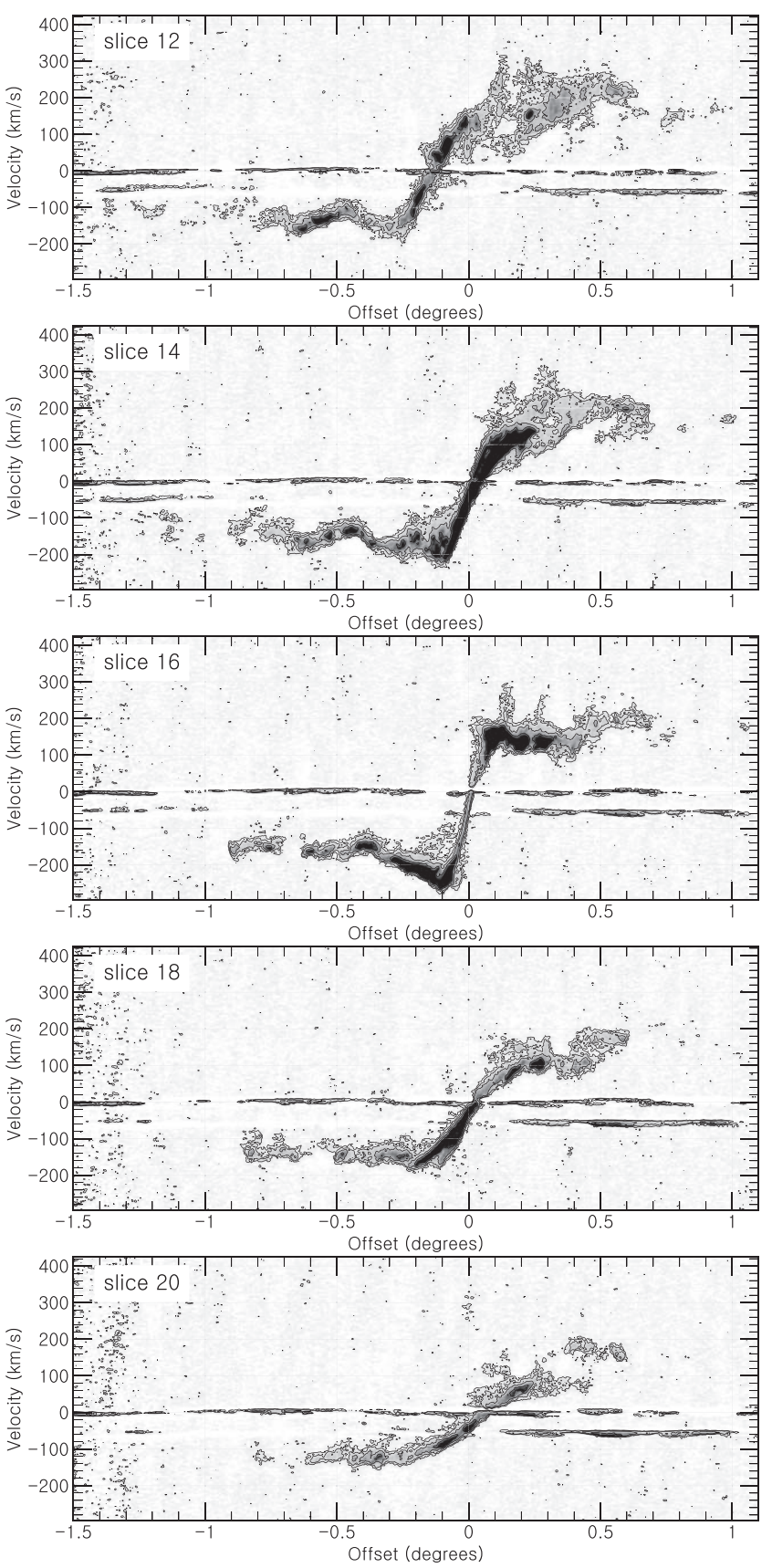

Figure 9. (Continued.)

combination of the smaller number of baselines available (a third of the telescopes had already transitioned to EVLA status and were not used) and the relatively large amount of RFI which necessitated a significant amount of flagging. We tried combining these data with our VLA mosaic to produce one combined data set, but this produced inferior results due to the irregular pointing grid and varying noise levels in the overlap region.

The higher noise level and presence of residual RFI artefacts in the data means we used an alternative method to create an unbiased integrated intensity map. All $\mathrm{HI}$ in the observed region was constrained to the velocity range from -120 to $-80 \mathrm{~km} \mathrm{~s}^{-1}$ and we therefore only considered the channels in this velocity range. These were spatially smoothed to twice the original beam size. We selected all signal above $3 \sigma$ (smoothed) per channel and also present in at least two consecutive channels. The resulting mask was applied to the original resolution data cube, and from the latter a zeroth-moment map was created. These data are discussed further in Section 3.4.

\section{Discussion of the Data}

\subsection{Moment Maps and Position-velocity Slices}

The zeroth-moment map (Figure 3) shows features not visible in the Yun et al. $(1994,2000)$ data, such as the full length of the arm between M81 and NGC 2976, emission 


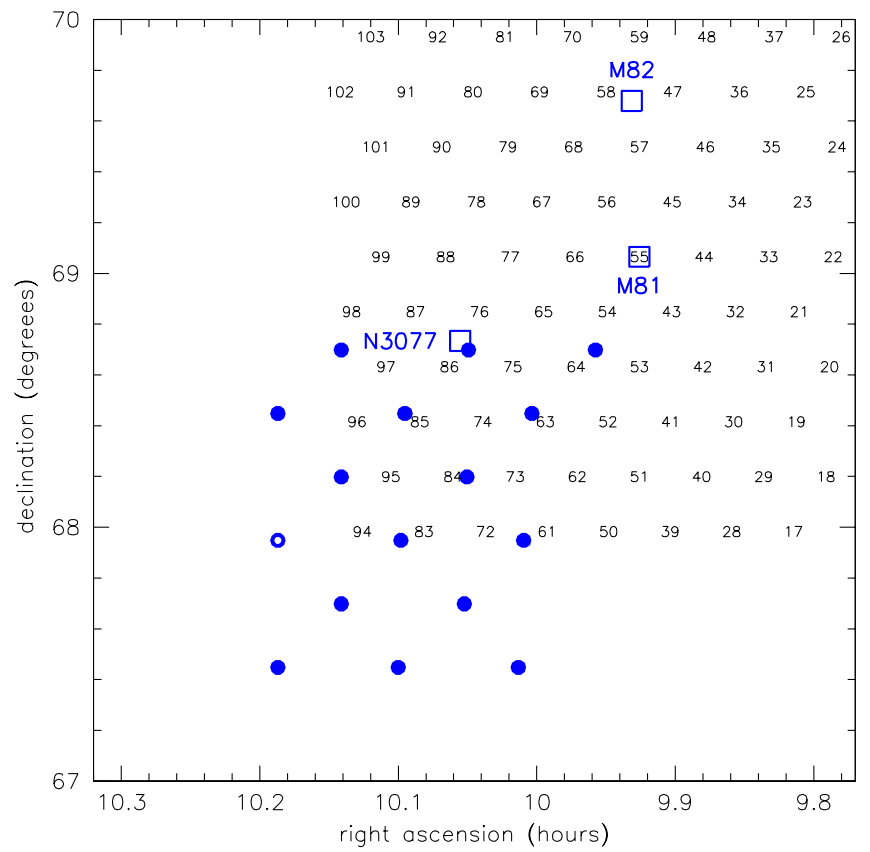

Figure 10. Central positions of the pointings of the AW683 mosaic compared with our mosaic. Filled blue circles indicate the pointings used here. The blue open circle shows the position of a pointing observed but not used. Other numbers and symbols are as in Figure 1.

between NGC 2976 and M81, and the presence of clouds to the $\mathrm{SE}$ of the triplet. The existence of the northern part of the NGC 2976 arm was already known from observations by Appleton et al. (1981) and Appleton \& van der Hulst (1988), as well as from the 24-pointing mosaic by Yun et al. (2000). The zerospacing corrected moment map convincingly shows that this arm splits, with one part extending down to NGC 2976, as was also shown in the GBT observations in Chynoweth et al. (2008). Also visible close to the northernmost edge of the mosaic is dwarf galaxy M81dwB (UGC 5423) at $10^{\mathrm{h}} 05^{\mathrm{m}} 30^{\mathrm{s}}$, $+70^{\circ} 21^{\prime} 52^{\prime \prime}$.

One striking result is that the observed area away from the triplet is mostly empty. We do not find a large population of small H I clouds that are not associated with the tidal features, even though the $5 \sigma \mathrm{HI}$ mass limit for an unresolved cloud is $\sim 10^{4} M_{\odot}$ for a velocity width of $\sim 10 \mathrm{~km} \mathrm{~s}^{-1}$. Even taking into account that clouds may be resolved by a few beams, or have velocity widths that are a factor of few larger, this still implies upper limits below $\sim 10^{5} M_{\odot}$ for a hypothetical population of free-floating $\mathrm{HI}$ clouds. It is often thought that these freefloating clouds could be embedded in mini-dark-matter halos, with implications for cosmological problems such as the "missing satellites" problem (e.g., Kauffmann et al. 1993). A more extensive discussion on cloud masses is given in Section 3.4.

The velocity field of M81 (Figure 4) shows a regularly rotating inner disk. The outer disk is more disturbed. The transition occurs at approximately the Holmberg radius. The largest deviations from regular rotation occur to the east of the center, along the minor axis, and are visible as strong kinks in the velocity contours. This region corresponds to the location of dwarf galaxy Holmberg IX. This is also visible in the position-velocity slices in Figure 9. Slice 10 and 11 cross this location, and the presence of the extra $\mathrm{H} \mathrm{I}$ is clearly visible at an offset of $\sim-0.1$.
The orientation of the kinematical minor axis of M82 seems to be almost perpendicular to its optical minor axis. It is likely that this is caused by the gas outflows in M82 (e.g., Yun et al. 1993b; Walter et al. 2002b; Leroy et al. 2015; Martini et al. 2018) affecting the velocity field. Slices 6 and 7 in Figure 9 show that in these regions $\mathrm{HI}$ is present with a velocity spread of close to $400 \mathrm{~km} \mathrm{~s}^{-1}$.

NGC 3077 is hardly visible kinematically, and the dynamics of the gas in that region are dominated by the interaction. It also does not stand out in slices 1-5 (Figure 9) which cross this area.

Note that the smaller clumps and stream fragments surrounding the main body of the triplet all have velocities close to those of the nearby parts of the triplet, indicating they are probably all associated with the observed tidal features.

The second-moment map (Figure 5) shows a north-south gradient in velocity dispersion, with lower values of around $5-10 \mathrm{~km} \mathrm{~s}^{-1}$ mainly found toward the southwest, while high values of $20 \mathrm{~km} \mathrm{~s}^{-1}$ and higher are found toward the northeast. Many of these high values are associated with M82, and inspection of the data cube shows that this is indeed diffuse gas that is spread over a large range in velocity, as shown by slices 6-8 (Figure 9).

The situation is different in the northern part of M81 and the connection with M82. Here the high values indicate the presence of multiple components at different velocities along the line of sight. This explains the extremely high secondmoment values of $>100 \mathrm{~km} \mathrm{~s}^{-1}$ found about $10^{\prime}$ to the north of the center of M81. Here, multiple, separate components with a maximum separation of $\sim 260 \mathrm{~km} \mathrm{~s}^{-1}$ are present. Slices $12-14$ (Figure 9) show this region at offsets between $\sim+0^{\circ} .1$ and $\sim+0.3$.

To disentangle these multiple components, most likely different physical structures along the same line of sight, requires a full $3 \mathrm{D}$ structural and kinematic model of all the $\mathrm{H}$, both the rotating disk of M81 and the various tidal filaments wrapping around M81 and its satellite galaxies. Athough the features just discussed are the most prominent, similar structures can be found at many places within the group; see, e.g., slice 9 at -0.4 and slice 7 at $0^{\circ} .0$.

Some of the high second-moment value clumps seen in the bridge between M81 and NGC 3077 are caused by H I clouds at different velocities from the main $\mathrm{H}$ I bridge features. These clouds are in the tidal structures, well away from the main galaxies. In contrast, the high values in the immediate proximity of NGC 3077 are intrinsic again, and indicate the presence of a gas component spread over a large range in velocity, as shown by the feature in slice 4 (Figure 9) at $\sim-0.55$.

In addition to the larger-scale phenomena described above, several interesting individual smaller-scale features can be made out in the position-velocity slices. One example is the high-velocity feature visible in slice 16 at an offset of +0.14 with anomalous velocities of up to $\sim 100 \mathrm{~km} \mathrm{~s}^{-1}$. It is located in the interarm region just south of the inner of the two prominent northern H I spiral arms of M81. Ultraviolet Galaxy Evolution Explorer (GALEX) (Gil de Paz et al. 2007) and $\mathrm{H} \alpha$ (Greenawalt et al. 1998) data, as well as the stellar density map discussed in Section 4.1, show the presence of star formation in the area, and it is likely that the feature is associated with a recent star formation event. Several similar, but less prominent, features are visible in the same area. 


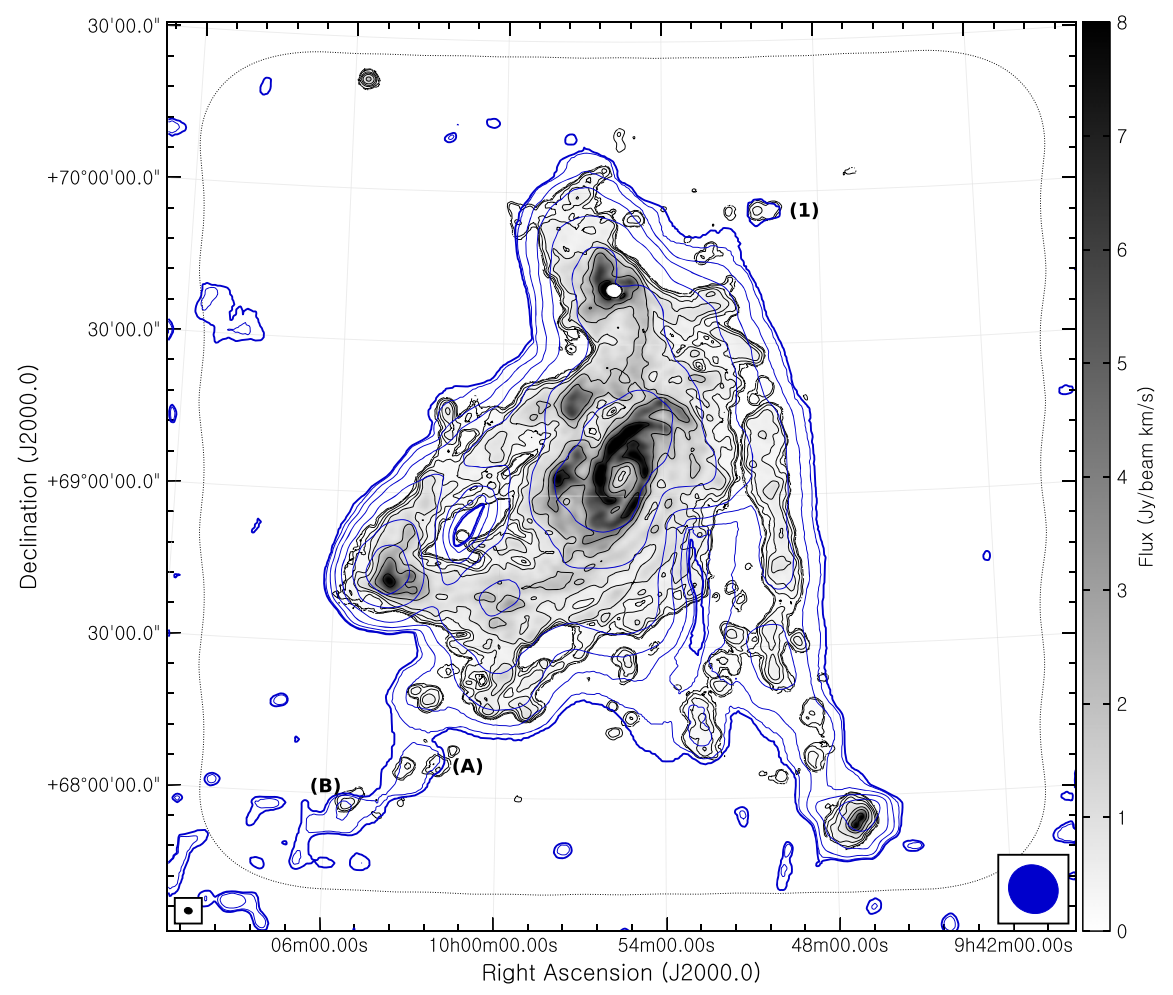

Figure 11. Comparison of our natural-weighted D-array zeroth-moment map with the GBT zeroth-moment map from Chynoweth et al. (2008). The D-array data are shown as grayscale and black contours, the GBT data as dark-blue contours. The grayscale runs from 0 (white) to 8 (black) Jy beam ${ }^{-1} \mathrm{~km} \mathrm{~s}^{-1}$. The GBT contour levels are shown at 1500 (thick contour), 3000, 7500, 15,000,30,000, 75,000, 150,000 and 300,000 kJy beam ${ }^{-1} \mathrm{~km} \mathrm{~s}^{-1}$ which corresponds to (4.5, 9.0, 22.5, 45, 90, $225,450,900) \times 10^{18} \mathrm{~cm}^{-2}$. The D-array mosaic contour values were chosen to have the same column densities, and are shown at $0.0329 \times(1,2,5,10,20,50,100$, 200) Jy beam ${ }^{-1} \mathrm{~km} \mathrm{~s}^{-1}$. The full GBT survey area is shown. The mosaic $50 \%$ sensitivity contour is shown as the dotted curve. The VLA beam is indicated in the bottom-left corner, the GBT beam in blue in the bottom-right corner. Numbers and letters indicate the cloud complexes described in Sections 3.2 and 3.4 .

\subsection{Comparison with GBT Data}

As noted in Section 1, the survey area presented here was also observed with the GBT, as published in Chynoweth et al. (2008). In Section 2.7 we described using the GBT data to correct for the missing spacings in the VLA data. As the zerospacing corrected cube is a combined data set with the resolution of the interferometry data and the flux of the singledish data, it in principle contains no new information that is not already present in the two source data sets. It is therefore instructive to compare these original data sets to get a understanding of where the various features visible in the moment maps originate.

Figure 11 displays an overlay of the Chynoweth et al. (2008) data on top of our D-array mosaic. The GBT beam size is $10 ! 1 \times 9 ! 4$, with a major axis position angle of $53^{\circ}$. This translates to a physical size of $10.7 \times 9.9 \mathrm{kpc}$.

The column density sensitivities of both data sets are similar. Chynoweth et al. (2008) quote a $1 \sigma, 1$ channel $\left(5.2 \mathrm{~km} \mathrm{~s}^{-1}\right)$ sensitivity of $2.5 \times 10^{17} \mathrm{~cm}^{-2}$. Smoothing our D-array data to the same velocity resolution yields a sensitivity of $3.5 \times$ $10^{17} \mathrm{~cm}^{-2}$. In Figure 11, we therefore chose identical contour levels for both data sets. We see a good correspondence between the HI distribution as observed by the VLA and the GBT. The only major discrepancy is immediately to the south-west of M81, where the GBT data show an extended north-south trough that is not visible in the VLA data. This trough is artificial and entirely due to the interpolation over the blanked Galactic emission that was used in the Chynoweth et al. (2008) paper to construct the moment map.
The low-column density filament seen in the GBT data near $10^{\mathrm{h}} 06^{\mathrm{m}},+68^{\circ} 00^{\prime}$, which is resolved into clumps with the VLA, extends to the edge of the GBT survey area, suggesting there may be additional $\mathrm{H}$ I clouds beyond the VLA survey area. We will return to this in Section 3.4.

The feature in the GBT data located near $10^{\mathrm{h}} 11^{\mathrm{m}},+69^{\circ} 30^{\prime}$ has a velocity of $\sim-110 \mathrm{~km} \mathrm{~s}^{-1}$ as detected in the original GBT data cube. At this position and velocity it is also marginally visible in the VLA mosaic. It is not included in the VLA moment map as its peak flux is below $3 \sigma$ and its location close to the $50 \%$ sensitivity contour makes identification more uncertain based on the VLA data alone.

The reverse situation is true for M81 Dw B (UGC 5423), a dwarf galaxy which is clearly detected in the VLA mosaic (at $\left.10^{\mathrm{h}} 05^{\mathrm{m}} 30^{\mathrm{s}},+70^{\circ} 21^{\prime} 52^{\prime \prime}\right)$, but is not visible in the GBT moment map. Inspection of the GBT data cube shows a marginal detection at the correct position and velocity, but it is located in the edge region of the GBT map where the noise is enhanced and many artificial features of similar extent and brightness are present.

It is striking that, especially toward the south, the lowcolumn density arms and streams detected by the GBT break up in clouds and clumps as observed by the VLA. An interesting question is whether these clouds represent all the H I seen in the lower-resolution GBT data, or whether they form the high column density tip of the iceberg in a surrounding lower column density component.

To address this, we compare the H I masses of a number of these clouds, selecting only objects that are far enough away spatially and spectrally from bright $\mathrm{HI}$ emission that may affect 
the object fluxes. As noted above, we consider the VLA and GBT data sets separately to better trace the origin of emission features. The zero-spacing corrected data is (for individual lowflux objects) less suited due to the various contributions from, among others, flux scale factors, masking and difference in velocity resolution that are difficult to quantify.

One example of a low-mass $\mathrm{H}$ I cloud is the isolated cloud to the northwest of M82, which Chynoweth et al. (2008) denote as "Cloud 1" (indicated as "1" in Figure 11). We find an H I mass of $3.2 \cdot 10^{6} M_{\odot}$, which is a factor 4.6 less than found by Chynoweth et al. (2008). (The other clouds discussed in that paper are affected by Galactic emission and therefore not discussed here.)

Other examples can be found to the south of the triplet. These are indicated in Figure 11 as "A" and "B." Complex A consist of two small clouds in the VLA data, and corresponds to single overdensity in the GBT map. Cloud B is a single cloud in the VLA data, corresponding with a single overdensity in the GBT map.

The two clouds A have a total mass of $4.8 \times 10^{6} M_{\odot}$. The mass of the corresponding GBT peak is $1.2 \times 10^{7} M_{\odot}$, or a factor of 2.5 higher. Cloud B has a mass of $3.2 \times 10^{6} M_{\odot}$ in the VLA data, and $1.2 \times 10^{7} M_{\odot}$ in the GBT map. This is a factor of 3.6 different. For completeness, we did check the combined data, and for the HI clouds discussed here found masses intermediate to the GBT and VLA masses.

In these particular comparisons we can be confident that the GBT is detecting excess H I not seen in the VLA data. This indicates that the low-column density filaments seen in the GBT data are not simply the VLA H I clouds observed at low resolution, but that they consist of substantial amounts of low-column density $\mathrm{HI}$ in which the clouds are embedded.

\subsection{Comparison of H I Masses: GBT versus VLA}

\subsubsection{Total H I Mass}

The previous section established that some of the isolated clouds seen in the VLA data are embedded in a low-column density H I component detected by the GBT. We can check if this is more generally the case by comparing the respective total $\mathrm{H}$ I masses found in both data sets. As discussed above, we compare the individual VLA and GBT sets, rather than the zero-spacing corrected data.

We use the moment maps to determine the total H I mass detected in the mosaic area. For the VLA C $+\mathrm{D}$ naturalweighted data we find a total flux of $2234.4 \mathrm{Jy} \mathrm{km} \mathrm{s}^{-1}$. Using the assumed distance of $3.63 \mathrm{Mpc}$, this gives a total H I mass of $6.94 \times 10^{9} M_{\odot}$.

The D-array data gives a slightly higher value of $2489.0 \mathrm{Jy} \mathrm{km} \mathrm{s}^{-1}$. This translates into an $\mathrm{HI}$ mass of $7.74 \times 10^{9} M_{\odot}$. These values are $\sim 35 \%$ higher than the total H I masses given in Yun (1999) and Appleton et al. (1981). This discrepancy is likely due to a combination of different survey volumes, column density sensitivities and Galactic foreground corrections. We show below that the latter alone can already amount to differences of $\sim 30 \%$ in the total fluxes.

For the GBT data of the M81 triplet, Chynoweth et al. (2008) report a total HI mass of $10.46 \times 10^{9} M_{\odot}$. This is substantially higher than the previous literature values, but also $\sim 35 \%$ higher than the value derived from our D-array data.

Chynoweth et al. (2008) note that their data were affected by Galactic foreground emission between -85 and $+25 \mathrm{~km} \mathrm{~s}^{-1}$. They

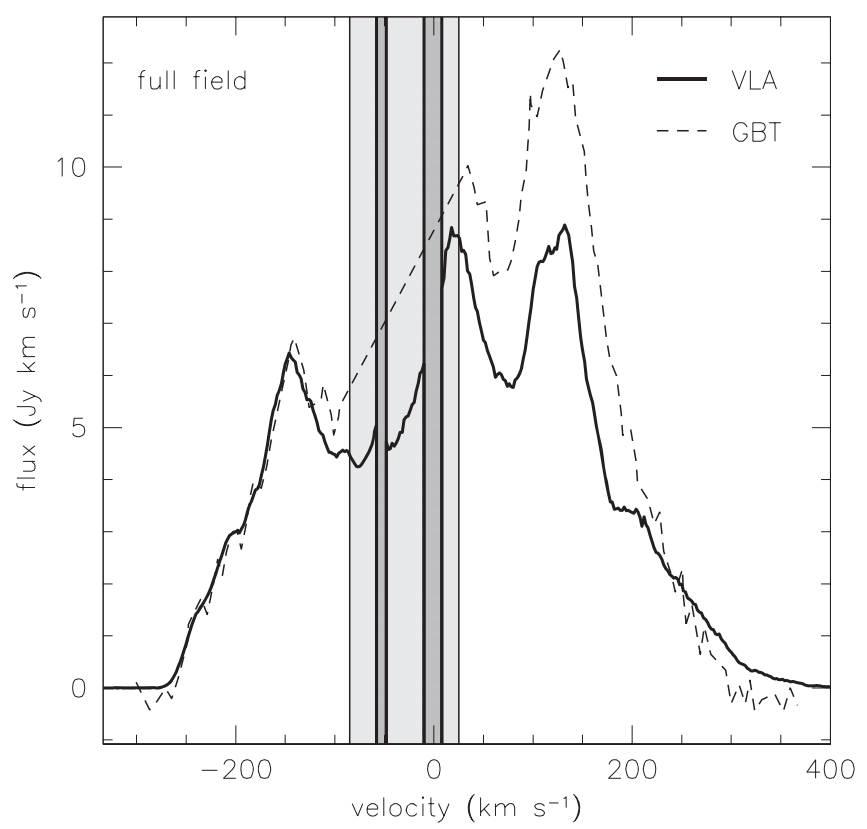

Figure 12. Comparison of integrated intensity profiles of the observed area. The thick full profile shows the integrated flux based on our D-array mosaic. The thin dashed profile show the integrated flux derived from the Chynoweth et al. (2008) GBT observations. The light-gray area indicates the velocity range over which Chynoweth et al. (2008) have interpolated their data. The dark-gray areas indicate the velocity ranges which we omitted from our data due to the Galactic emission. Note the different behavior of the profiles at positive velocities, probably indicating the presence of diffuse gas associated with M82.

replaced the data in these velocity channels with a linear interpolation based on the channels immediately adjacent to this range. From the global HI profile of the full area as shown in Figure 2 of Chynoweth et al. (2008), and also reproduced in Figure 12, we find that this interpolated part of the spectrum constitutes $29 \%$ of total flux they report.

The higher velocity resolution of our data allows us to gauge the accuracy of this correction. We overplot the global profiles of the full mosaic area in Figure 12. The D-array fluxes in the interpolated region of the GBT spectrum are $\sim 30 \%$ lower than the GBT interpolations. It is, however, not trivial to correct the GBT H I mass on the basis of this. Figure 12 shows that at negative velocities the GBT and D-array fluxes agree very well with each other, whereas at positive velocities the GBT has detected substantially more flux than the D-array. Note that, as discussed in Section 2.3, the behavior of detected flux as a function of clean depth is identical for channel maps with positive and negative velocities, so that the difference is not due to different relative importance of uncleaned flux in these channel maps.

In other words, at negative velocities the D-array observations have managed to detect almost all of the H I flux (mostly associated with the southern part of M81), while the extra GBT flux at positive velocities (associated with the very northern part of M81, with M82, and with the transition region in between) indicates the presence of an extended low column density H I component that is not present in the southern part of the triplet. Figure 12 shows that the difference between the integrated spectra is largest around the peak at $\sim 125 \mathrm{~km} \mathrm{~s}^{-1}$, and the "missing" gas is thus most likely associated with the already detected diffuse HI around M82. For a full synthesis observation, the largest angular scale the VLA is sensitive to at $1.4 \mathrm{GHz}$ is $\sim 16^{\prime}$, while for a single snapshot observation this 
is $\sim 8^{\prime}$. This range of scales is mostly larger than the GBT beam. So while the length of the integration time per pointing may have some influence on the recovery of structures larger than $\sim 10^{\prime}$, it is more likely that the difference between the integrated spectra is due to surface brightness sensitivity limitations.

We also considered the integrated spectrum of the zerospacing corrected $\mathrm{C}+\mathrm{D}$ data, and found a good match with the GBT profile at positive velocities. However, at negative velocities this profile significantly overestimates the flux compared to the GBT profile. The situation is reversed when using D-array corrected data. We tested the combination using different velocity ranges to determine the scale factor, but found this did not affect the outcomes. Due to the uncertainty in relative flux scales of these combined data, we therefore do not consider the zero-spacing corrected data further in this context. A full study of the relative fluxes found in the VLA and GBT data as a function of resolution is beyond the scope of this paper.

The presence of extra $\mathrm{HI}$ at positive velocities and its absence at negative velocities, with the transition happening exactly in the region affected by Galactic emission, makes deriving a more accurate correction for Galactic foreground correction difficult. Figure 12 suggests that, with various extra components and corrections canceling each other, the total $\mathrm{H} \mathrm{I}$ mass estimate given in Chynoweth et al. (2008) is probably an overestimate, but likely by not more than $\sim 5 \%-10 \%$. Taking all this into account, we can therefore conclude that the GBT data show the presence $\sim 25 \%-30 \%$ more H I than our VLA D-array mosaic.

\subsubsection{H I Masses of the Triplet Galaxies}

The H I masses of the major triplet galaxies are more difficult to determine and compare, as the extent of their H I disks cannot be well determined due to the presence of the tidal H I component. Chynoweth et al. (2008) compare the H I masses of the three major triplet galaxies as derived from the GBT data, the Yun (1999) data and the Appleton et al. (1981) data. The last are also based on single-dish data.

Appleton et al. (1981) define the H I masses of the galaxies as the mass measured within the Holmberg ellipse of the respective objects and Chynoweth et al. (2008) follow that definition. As noted by Appleton et al. (1981), this choice of radius likely underestimates the $\mathrm{HI}$ masses. In M81 the Holmberg radius only encompasses the inner, high-density spiral arms; in M82 it misses much of the extra-planar gas, while in NGC 3077 the main H I component falls outside the Holmberg radius. Nevertheless, in the absence of any clear physical indicators, other choices would be equally arbitrary.

We here apply the same procedure to our D-array data, using the parameters given in Table 1 of Appleton et al. (1981). To get an estimate of the uncertainty in the masses, we also derive the H I masses within a radius of $2 R_{25}$, with the $R_{25}$ values taken from HyperLEDA, but still adopting the orientations given in Appleton et al. (1981). Larger radii are impractical as the disks of M81 and M82 start overlapping at $\sim 2.5 R_{25}$. The masses are listed in Table 3 and compared with the Chynoweth et al. (2008), Yun (1999), and Appleton et al. (1981) masses. For M81, an alternative definition for the H I mass could be made by using the transition radius between the ordered motion of the inner disk and the more disturbed motion beyond that (see the velocity field in Figure 4). This radius turns out to be
Table 3

Comparison of H I Masses

\begin{tabular}{lccccc}
\hline \hline Galaxy & $\begin{array}{c}M_{\mathrm{H} \mathrm{I}} \\
(\mathrm{Ho})\end{array}$ & $\begin{array}{c}M_{\mathrm{H} \mathrm{I}} \\
\left(2 R_{25}\right) \\
\left(\times 10^{9} M_{\odot}\right)\end{array}$ & $\begin{array}{c}M_{\mathrm{H} \mathrm{I}} \\
(\mathrm{Ch} 08)\end{array}$ & $\begin{array}{c}M_{\mathrm{H} \mathrm{I}} \\
(\mathrm{Y} 99)\end{array}$ & $\begin{array}{c}M_{\mathrm{H} \mathrm{I}} \\
(\mathrm{Ap} 81)\end{array}$ \\
\hline M81 & 2.29 & 2.79 & 2.67 & 2.81 & 2.19 \\
M82 & 0.44 & 0.75 & 0.75 & 0.80 & 0.72 \\
NGC 3077 & 0.23 & 0.31 & 1.01 & 0.69 & 1.00 \\
Total $^{\mathrm{a}}$ & 7.74 & $\ldots$ & 10.46 & 5.6 & 5.4 \\
\hline
\end{tabular}

Notes. (Ho): H I mass within Holmberg radius from D-array mosaic; $\left(2 R_{25}\right)$ : H I mass within $2 R_{25}$ radius from D-array mosaic; (Ch08): H I mass from Chynoweth et al. (2008); (Y99): H I mass from Yun (1999); (Ap81): H I mass from Appleton et al. (1981).

${ }^{a}$ Value refers to entire observed area.

almost exactly equal to the Holmberg radius, so this mass is equal to that already listed in Table 3.

There is a large spread in H I mass values for each galaxy. We have already established that Galactic foreground corrections introduce an extra uncertainty in the Chynoweth et al. (2008) data, mostly due to the lower velocity resolution. It is likely that a similar uncertainty applies to the Yun (1999) and Appleton et al. (1981) masses as well. A full and proper determination of the "true" $\mathrm{HI}$ masses of the three main galaxies would thus require a further in-depth analysis and comparison of all these effects.

\subsection{The Southeast Clouds}

In Section 3.2, we compared the H I masses of the overdensities "A" and "B" seen in the GBT map in Figure 11. These overdensities correspond with a number of more compact clumps as observed with the VLA.

The GBT map also shows that the H I filament containing the overdensities extends all the way to the SE corner of the observed area. Our VLA mosaic does not extend this far, but we can use additional observations to explore this area at higher resolution. We use the data from project $\mathrm{AW683}$, which consists of a 16-pointing mosaic observed in C- and D-array and partly overlapping with the SE corner of our mosaic. A description of these data is given in Section 2.9.

Inspection of the AW683 data cube clearly shows the presence of clouds A and B. We show the zeroth-moment map derived from these data in Figure 13, in combination with the corresponding maps from the GBT and our mosaic. It is clear that the low column density structure detected by the GBT at the edge of the survey area coincides with a clump of $\mathrm{H}$ I in the extended area mosaic. For this clump, we find a mass of $6.3 \times 10^{6} M_{\odot}$, comparable to that of the A and B clumps.

The velocities of these clumps are all close to that of the more prominent $\mathrm{HI}$ features in this general area, suggesting that they are tidal debris from the triplet interactions. There are no other new H I clumps of comparable flux in this area. We find a number of marginal detections of smaller clumps, but deeper observations will be needed to confirm whether these are real.

The H I masses of the SE clumps are larger than those of the smallest dwarf galaxies that have been detected in $\mathrm{HI}$ in the Local Volume. An example is Leo $\mathrm{P}$, a low-mass, gasdominated galaxy with an H I mass of $8.1 \times 10^{5} M_{\odot}($ McQuinn et al. 2015). It, and other galaxies like it, are known to contain dark matter (Bernstein-Cooper et al. 2014), and potentially 


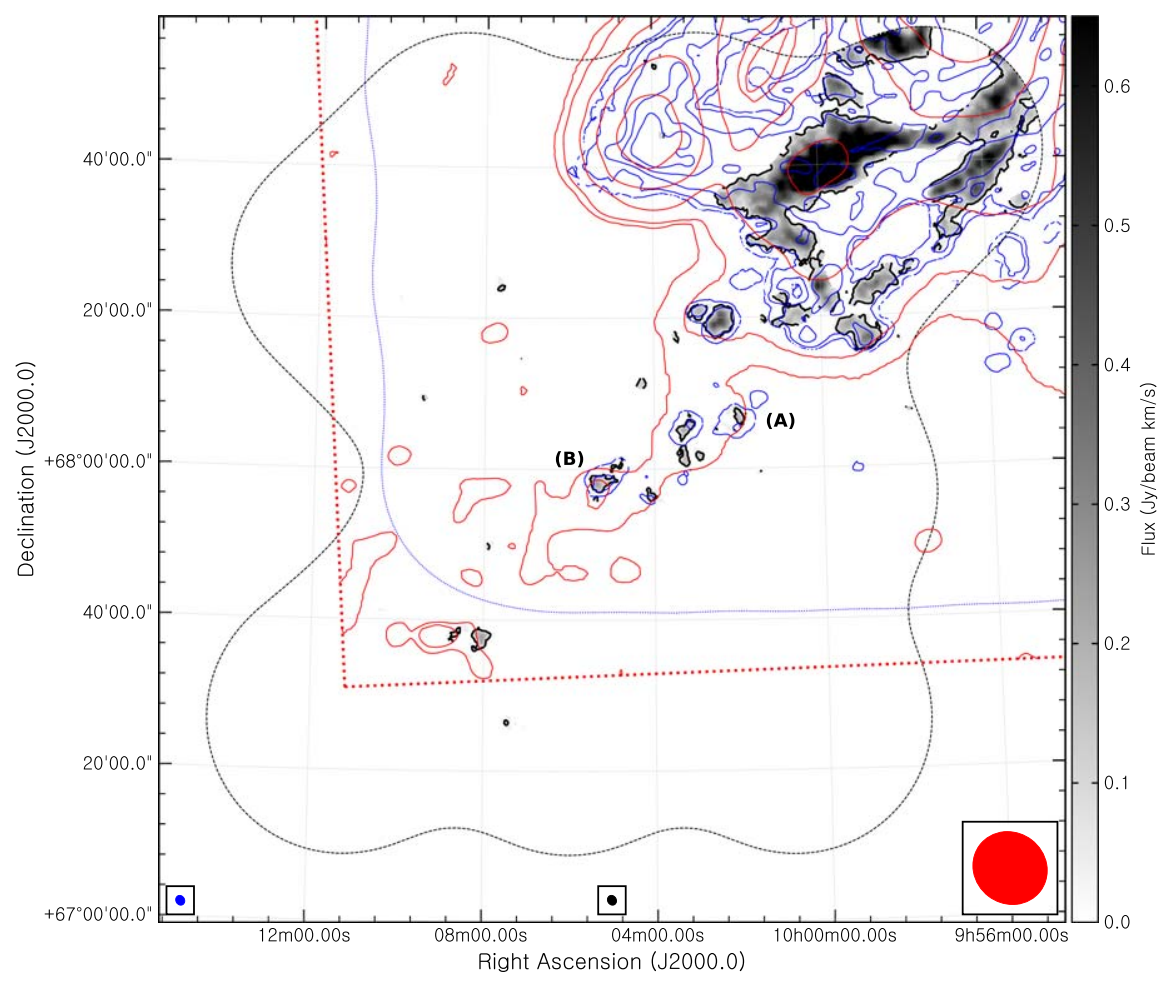

Figure 13. Comparison of emission detected in the D-array AW683 mosaic, the GBT observations, and our D-array mosaic. The grayscale shows the emission detected between -120 and $-80 \mathrm{~km} \mathrm{~s}^{-1}$ in the AW683 mosaic, and runs from 0 (white) to 0.65 (black) Jy beam ${ }^{-1} \mathrm{~km} \mathrm{~s}^{-1}$. The corresponding black contour shows the $1.2 \times 10^{19} \mathrm{~cm}^{-2}$ column density level. Red and blue contours show the GBT and VLA emission at identical repective column density levels of (4.5, 22.5, 90, 225, $450) \times 10^{18} \mathrm{~cm}^{-2}$ (cf. Figure 11). The different H I distribution in the M81-NGC 3077 bridge is due to the different velocity ranges displayed. The blue dotted curve indicated the 50\% sensitivity level of our mosaic, the black curve that of the 15-pointing AW683 mosaic. The red dashed lines show the extent of the GBT survey area. Beams are indicated at the bottom using the respective contour colors. The letters indicate the cloud complexes described in Section 3.4.

could help solve some of the problems that exist in small-scale $\Lambda \mathrm{CDM}$, such as the "missing satellites" problem (see, e.g., Kauffmann et al. 1993 for an early discussion). Similarly, ultracompact high-velocity clouds (UHVCs), which have similar H I masses and sizes as the SE clouds, are thought to be candidate low-mass galaxies harbouring dark matter halos (Adams et al. 2016).

An interesting question to explore is whether the SE clumps have properties consistent with low-mass galaxies or UHVCs. We extract integrated velocity profiles of clouds A and B from the D-array cube, where we treat cloud A as two separate objects, hereafter "A east" and "A west." We use the D-array zeroth-moment map as a mask to define the area over which to extract the profiles. We extract profiles from both the masked cube (used to create the moment maps) and the unmasked cube. The three sets of profiles are shown in Figure 14. The profiles are narrow and well-defined. For the A-clouds, the masked and unmasked profiles agree well with each other. For the B-cloud, the unmasked profile is significantly wider than the masked profile. Inspection of the cube shows that this extra emission is due to a nearby diffuse $\mathrm{HI}$ feature, though it is not clear whether this emission belongs to the cloud (and is extra confirmation that these clouds do not exist in isolation).

We measure the velocity widths $W_{20}$ and $W_{50}$ of the masked and unmasked profiles at $20 \%$ and $50 \%$ of the peak flux. These are listed in Table 4 . We use these widths to calculate indicative dynamical masses $M_{\text {dyn }} \sin i=(W / 2)^{2} R / G$ of these clouds, where $W$ is the velocity width $W_{20}$ or $W_{50}, R$ is the radius of the cloud, $G$ is the gravitational constant, and $i$ the inclination of the cloud. For the radius we simply use half of a cloud's extent along its major axis. These radii are also listed in
Table 4, along with the resulting indicative dynamical masses. These values assume the clouds are fully gravitationally (rotationally) supported. Other estimates for the dynamical mass, which assume that the clouds are fully or partially pressure supported, tend to give higher values for $M_{\mathrm{dyn}}$, so the values given in Table 4 are lower limits.

Comparing the HI masses with the indicative dynamical masses (we find no evidence for a stellar component in SDSS images), we find that the dynamical to $\mathrm{H} \mathrm{I}$ mass ratios are high: for the $W_{20}$ values we find an average ratio of $59.8 \pm 21.7$ (where we have omitted the value of the unmasked B-cloud profile), and for the $W_{50}$ values we find a ratio of $23.7 \pm 11.4$.

Using the GBT HI masses instead would decrease these ratios by a factor $\sim 3$ (see Section 3.4), but this would still give ratios substantially larger than unity.

At first glance, these results would indicate that the clouds are dark-matter-dominated, and good candidates for low-mass galaxies. Indeed, the velocity widths, $\mathrm{H}$ I masses and dynamical masses are fully consistent with those of the UHVC candidates and the dwarf galaxies Leo $\mathrm{T}$ and $\mathrm{P}$, as described in Adams et al. (2016).

There is, however, one big difference. The clouds discussed here are not isolated, but part of a larger, tidal structure. In contrast, the UHVC candidates and Leo $\mathrm{T}$ and $\mathrm{P}$ galaxies are isolated. Interpreting their velocity width is therefore less ambiguous than for clouds $\mathrm{A}$ and $\mathrm{B}$, whose velocity widths could also be explained by processes other than rotation. For example, the tidal arm in which the clouds are embedded shows a north-south velocity gradient of $\sim 50 \mathrm{~km} \mathrm{~s}^{-1}$, and part of this gradient could be reflected in the clouds. Streaming motions of the gas in the arm are a possibility as well. Finally, as discussed 


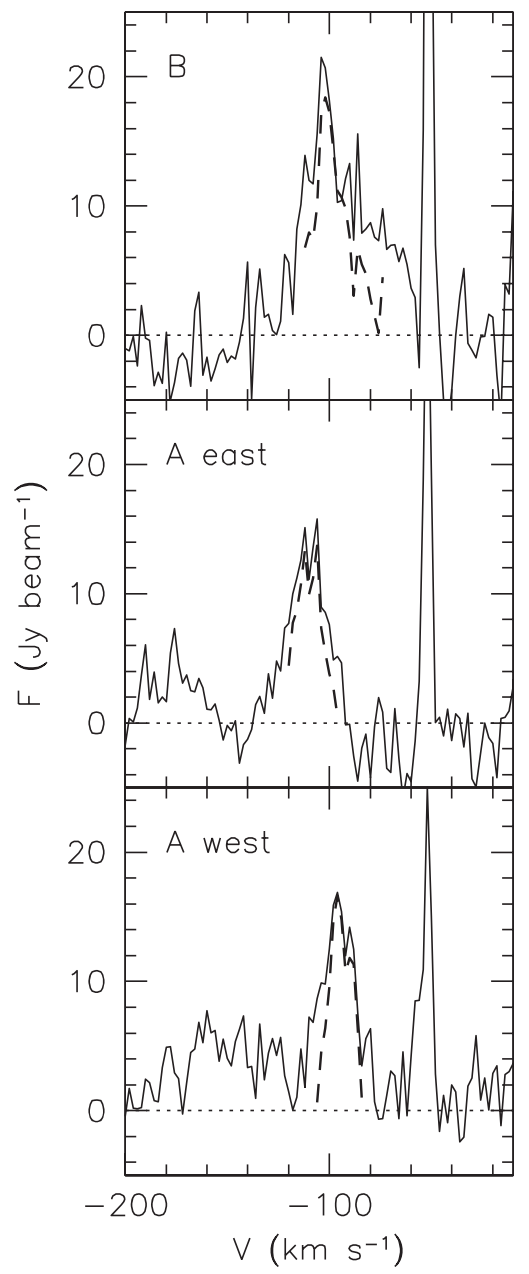

Figure 14. Velocity profiles of clouds A east, A west, and B, integrated over their area as shown in Figure 11. Full profiles are derived from the unmasked D-array cube, dashed profiles from the masked version. The peak at $\sim-50 \mathrm{~km} \mathrm{~s}^{-1}$ is due to Galactic foreground emission.

earlier, it is not clear whether the clouds are separate, isolated physical entities. More likely they are density enhancements in the tidal arm that could possibly evolve into tidal dwarfs.

The fact that the clouds are only observed in or near tidal structures, and not in the rest of the mosaic volume (which has after all been observed to the same depth and sensitivity), is another argument against the interpretation of the velocity widths as evidence for these clouds being dark-matter-dominated objects. We therefore find no unambiguous evidence for dark-matterdominated clouds in our survey volume.

\section{Star Formation, DLAs, and Velocity Dispersions}

\subsection{Star Formation in and around the M81 Triplet}

So far, we have discussed the $\mathrm{H}$ I distribution in and around the M81 triplet focusing on the dynamics, and implications for cloud masses and dark matter content. H I is, however, also strongly linked to star formation. For example, in many nearby galaxies, whenever the $\mathrm{H}$ I column density in the disk reaches a certain threshold, star formation is seen to occur (e.g., Skillman 1987). More recently, similar thresholds have been observed also in the outer disks of galaxies using deep $\mathrm{H} \alpha$ imaging (e.g., Ferguson et al. 1998) or GALEX ultraviolet observations (e.g., Bigiel et al. 2010).
Star formation is linked to H I through the molecular phase of the gas. Recent detailed studies of the molecular gas in the triplet galaxies show a large variety in the morphology and kinematics of this gas. CO observations of M82 show that the molecular gas extends far beyond the disk, with the wellknown gas outflows dominating the distribution (Leroy et al. 2015). The $\mathrm{CO}$ in M81 is much less prominent and mainly associated with the dust in the spiral arms (Brouillet et al. 1991; Sánchez-Gallego et al. 2011). The CO in NGC 3077 is discussed in Walter et al. (2002a) and Meier et al. (2001). As is the case with the H I, much of the molecular gas associated with NGC 3077 is found outside the optically bright part of the galaxy (Walter \& Heithausen 1999; Walter et al. 2006).

As for the stars themselves, the M81 triplet is close enough that its luminous stellar populations can be resolved using large ground-based telescopes (e.g., Barker et al. 2009; Mouhcine \& Ibata 2009; Okamoto et al. 2015). These studies find that the young stars closely follow the $\mathrm{H}$ I distribution as presented in Yun et al. (1994), while the older stars are found in extended, tidally disturbed halos, well beyond the $\mathrm{HI}$ distribution.

Here we re-examine this correlation using the new $\mathrm{HI}$ observations presented in this paper and deep resolved star photometry obtained with the Subaru $8 \mathrm{~m}$ telescope and the Suprime-Cam instrument (Miyazaki et al. 2002). This instrument consists of 10 CCDs of $2048 \times 4096$ pixels arranged in a $2 \times 5$ pattern, with a pixel scale of 0 ". 2 and a total field of view of approximately $34^{\prime} \times 27^{\prime}$ (including long edge inter-chip gaps of 16-17 arcsec and short edge gaps of 5-6 arcsec). The data set consists of seven distinct pointings with Suprime-Cam, covering a total area of $\sim 1.8$ square degrees, with exposure times of $\sim 6300-7600 \mathrm{~s}$ in the Johnson $V$-band and $\sim 4300-4500 \mathrm{~s}$ in the Sloan $i^{\prime}$-band. The seeing was in the range 0 ". $7-1$ ". 1 . One field was obtained during the period 2005 January 7-8, while the remaining six fields were obtained during 2010 January 16-18. All data were acquired, processed, and calibrated onto Johnson-Cousins $V, I$ using the same set-up and procedure as the 2005 data, which were previously presented in Barker et al. (2009). Finally, following Bernard et al. (2012), point-spread function fitting photometry was performed on the individual exposures using the standalone version of the DAOPHOT/ALLSTAR/ALLFRAME suite of programs (Stetson 1994). Extinction corrections were applied to individual sources using the maps of Schlegel et al. (1998) and the coefficients provided by Schlafly \& Finkbeiner (2011) for an $R_{V}=3.1$ reddening law.

To isolate $\mathrm{OB}$ stars within the object catalog, we selected all high-quality stellar sources with extinction-corrected magnitudes $I_{0}<25.25$ and extinction-corrected colors $-1.0<$ $(V-I)_{0}<0.2$. As shown by Barker et al. (2009), these cuts isolate main sequence stars with ages of $10-150 \mathrm{Myr}$. To compensate for the worse seeing conditions during the observations of the central field covering the northern part of M81, leading to a lower completeness fraction in the selected color and magnitude ranges, we applied a small correction factor of 3.9 to the weighting scheme in this region. In order to facilitate a direct comparison with our H I data, we created an image of the young stellar distribution by representing each object with a Gaussian equal to the natural-weighted beam size of the $\mathrm{H}$ I data and scale the amplitude using the $V$-band brightness. This better illustrates the relative density and brightnesses variations of the young star population compared to simply plotting the individual star counts. 


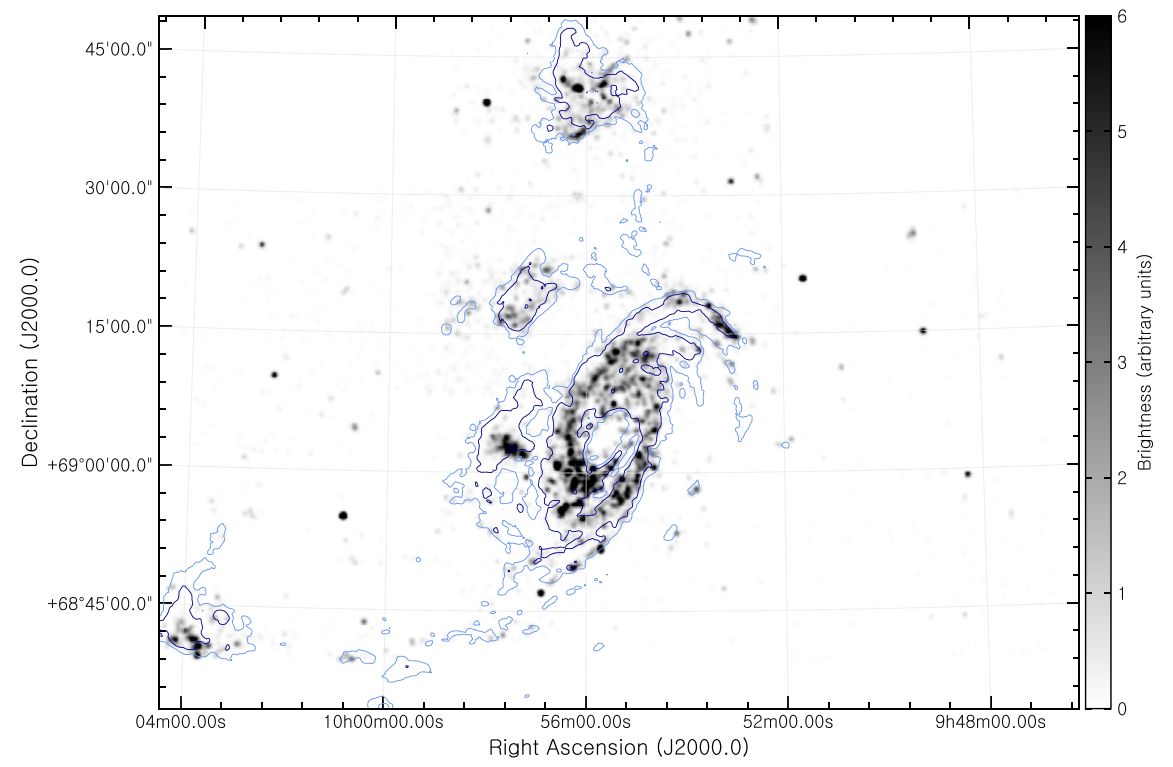

Figure 15. Luminosity-weighted distribution of OB stars from the Subaru Suprime-Cam catalog. Note the two stellar arcs above and below the M82 disk. Overplotted are the $3 \times 10^{20} \mathrm{~cm}^{-2}$ (light blue) and the $6 \times 10^{20} \mathrm{~cm}^{-2}$ (dark blue) H I column density contours.

Table 4

Comparison of Properties of H I Clouds

\begin{tabular}{|c|c|c|c|c|c|c|c|}
\hline Cloud & Type & $\begin{array}{c}M_{\mathrm{H} \mathrm{I}} \\
\left(\times 10^{6} M_{\odot}\right)\end{array}$ & $\begin{array}{r}R_{\mathrm{H} \mathrm{I}} \\
(\mathrm{kpc})\end{array}$ & $\begin{array}{c}W_{20} \\
\left(\mathrm{~km} \mathrm{~s}^{-1}\right)\end{array}$ & $\begin{array}{c}W_{50} \\
\left(\mathrm{~km} \mathrm{~s}^{-1}\right)\end{array}$ & $\begin{array}{l}M_{\mathrm{dyn}}^{W 20} \sin i \\
\left(\times 10^{6} M_{\odot}\right)\end{array}$ & $\begin{array}{l}M_{\mathrm{dyn}}^{W 50} \sin i \\
\left(\times 10^{6} M_{\odot}\right)\end{array}$ \\
\hline \multirow[t]{2}{*}{ A east } & masked & 2.0 & 2.4 & 25 & 15 & 87.2 & 31.4 \\
\hline & unmasked & 2.0 & 2.4 & 34 & 24 & 161.3 & 80.3 \\
\hline & unmasked & 2.8 & 3.1 & 32 & 20 & 184.5 & 72.1 \\
\hline \multirow[t]{2}{*}{ B } & masked & 3.2 & 3.3 & 36 & 17 & 248.6 & 55.4 \\
\hline & unmasked & 3.2 & 3.3 & $(58)^{\mathrm{a}}$ & 24 & 645.2 & 110.5 \\
\hline
\end{tabular}

Note.

${ }^{\mathrm{a}}$ This value is probably affected by emission not belonging to the cloud.

The luminosity-weighted distribution of OB stars is shown in Figure 15. An interesting feature is the pair of arcs that can be seen above and below the disk of M82. These arcs were also noted by Okamoto et al. (2015), and the southern arc was also discussed in detail in Davidge (2008) and Sun et al. (2005). Davidge (2008) estimated that the stellar mass of the arc is between $3 \times 10^{5} M_{\odot}$ and $2 \times 10^{6} M_{\odot}$, with an age of $\sim 5 \times 10^{7} \mathrm{yr}$, consistent with the typical interaction timescale in the triplet. Davidge speculated that the arc could have formed due to triggered star formation as a result of the M82 outflows and noted that it coincides with a similar arc seen in GALEX UV-data (Hoopes et al. 2005). A comparison of Figure 15 with our integrated H I map shows that the arc also coincides with a ridge in the H I surface density. The northern arc, while equally well defined in the young stars distribution, does however show no such corresponding features in the UV or HI.

A comparison of the stellar distribution in Figure 15 with the $\mathrm{HI}$ distribution in Figure 3 shows a close correspondence between the $\mathrm{OB}$ star concentrations and the majority of the $\mathrm{HI}$ peaks. Some peaks can still be identified that do not have corresponding star formation, but due to the timescale needed for star formation, one would not expect a one hundred percent match. This correspondence is of course a well-known result, first demonstrated in the early work by Skillman (1987), indicating that a minimum column density is needed for star formation. Much work has been done in trying to quantify and understand this star formation threshold. A full review of this field is beyond the scope of this paper, and we refer to the overview by Kennicutt \& Evans (2012). Estimates of the star formation threshold value usually arrive at values of a few times $10^{20} \mathrm{~cm}^{-2}$. A derivation on theoretical grounds is given in Schaye (2004), who argue for a value of $3-10 \times 10^{20} \mathrm{~cm}^{-2}$ over a range in gas fractions, metallicity, UV radiation intensity and amount of turbulence.

In Figure 15 , we overplot the $3 \times 10^{20} \mathrm{~cm}^{-2}$ contour on the young stellar distribution. Projection effects due to the geometry of the M81 triplet increase the observed surface densities from those that would be seen when face-on. Although it is difficult to correct for this for the triplet as a whole, we know that in the M81 disk, with an inclination of $57^{\circ}$, the observed surface densities are close to a factor of two higher than the face-on ones. We therefore also overplot the $6 \times 10^{20} \mathrm{~cm}^{-2}$ contour.

The latter contour tightly encloses the star formation in the main galaxies, while the lower $3 \times 10^{20} \mathrm{~cm}^{-2}$ contour also encompasses some of the fainter stars in the bridge between M81 and NGC 3077. Looking at the stellar luminosities we find that the $6 \times 10^{20} \mathrm{~cm}^{-2}$ contour encompasses $\sim 65 \%$ of the total OB star luminosity, while the $3 \times 10^{20} \mathrm{~cm}^{-2}$ contour contains $\sim 81 \%$ of the total OB star flux. These results are consistent with the 
findings of Barker et al. (2009), who examine the evidence for a star formation threshold by comparing the $\mathrm{HI}$ and $\mathrm{OB}$ star distribution in M81 over a significantly smaller area. The fact that the relation between the number and luminosities of OB stars and the H I column density holds for the entire field analyzed here suggests that star formation threshold is indeed a local phenomenon, independent of whether the gas and stars are in a disk environment or not.

\subsection{Comparison with DLAs}

Direct observations in emission of the cosmological evolution of $\mathrm{HI}$ in galaxies at high redshifts will probably stay outside our reach until the advent of future radio telescopes such as the Square Kilometre Array. Absorption line observations toward quasars, in contrast, are already giving us valuable clues about high-redshift HI. Much of the information is gleaned from absorption lines in Ly $\alpha$. These reveal the signature of many intervening $\mathrm{HI}$ clouds over a large range in column density. The detections with the highest column densities, $N_{\mathrm{H} \mathrm{I}}>2 \times 10^{20} \mathrm{~cm}^{-2}$, are known as DLAs (Wolfe et al. 1986; see also the review by Wolfe et al. 2005).

The DLAs are thought to probe the high- $z$ equivalents of gas disks observed in $21 \mathrm{~cm}$ at $z=0$ (Wolfe et al. 1995, 2005). Although many of their observed characteristics could be reproduced by simulations (e.g., Rahmati \& Schaye 2014), the single largest discrepancy was the inability to reproduce the observed distribution of velocity width measurements, quantified by the $\Delta V_{90}$ parameter. For these absorption observations, this parameter is defined as the difference between the velocities where the cumulative optical depth profile crosses the $5 \%$ and the $95 \%$ levels.

Early simulations obtained distributions peaking at low $\Delta V_{90}$ values of $\sim 50 \mathrm{~km} \mathrm{~s}^{-1}$ without the high velocity-width tail of $\Delta V_{90}>100 \mathrm{~km} \mathrm{~s}^{-1}$ found in the observations (e.g., Pontzen et al. 2008). This implied that more energy was needed to increase the gas velocities. The favored method of achieving this is through feedback (e.g., stellar winds, superwinds). Simulations which included post-hoc feedback showed that this could substantially increase the observed $\Delta V_{90}$ distribution (e.g., Tescari et al. 2009). Later simulations added in these feedback prescriptions and can now much better reproduce the observed $\Delta V_{90}$ distribution (e.g., Barnes \& Haehnelt 2014; Bird et al. 2015). The latter authors show that in the Illustris simulation, the largest $\Delta V_{90}$ objects are found to come from sightlines intersecting multiple individual $\mathrm{H}$ I structures.

If feedback does indeed play a major role in determining the velocity widths of the DLAs, then an interesting question is whether we can find low-redshift counterparts of the DLAs and study in detail the processes giving rise to the observed $\Delta V_{90}$ distribution. Using the $\mathrm{HI}$ emission data from the THINGS survey, Zwaan et al. (2008) derived the probability distribution function of $\Delta V_{90}$ of galaxies in the local universe. This can be directly compared with the DLA $\Delta V_{90}$ distribution if the lowion metals used to trace the DLAs are distributed like the neutral gas. Zwaan et al. (2008) argue that this is a reasonable assumption, though Prochaska et al. (2002) note that smallscale spatial variations in metallicity might affect this comparison. Like Prochaska et al. (2002), we here proceed under the assumption that these differences are not important to the kinematical properties we are studying here.

Zwaan et al. (2008) find that the distribution of $\Delta V_{90}$ for the THINGS galaxies is sharply peaked around $\sim 30 \mathrm{~km} \mathrm{~s}^{-1}$, a

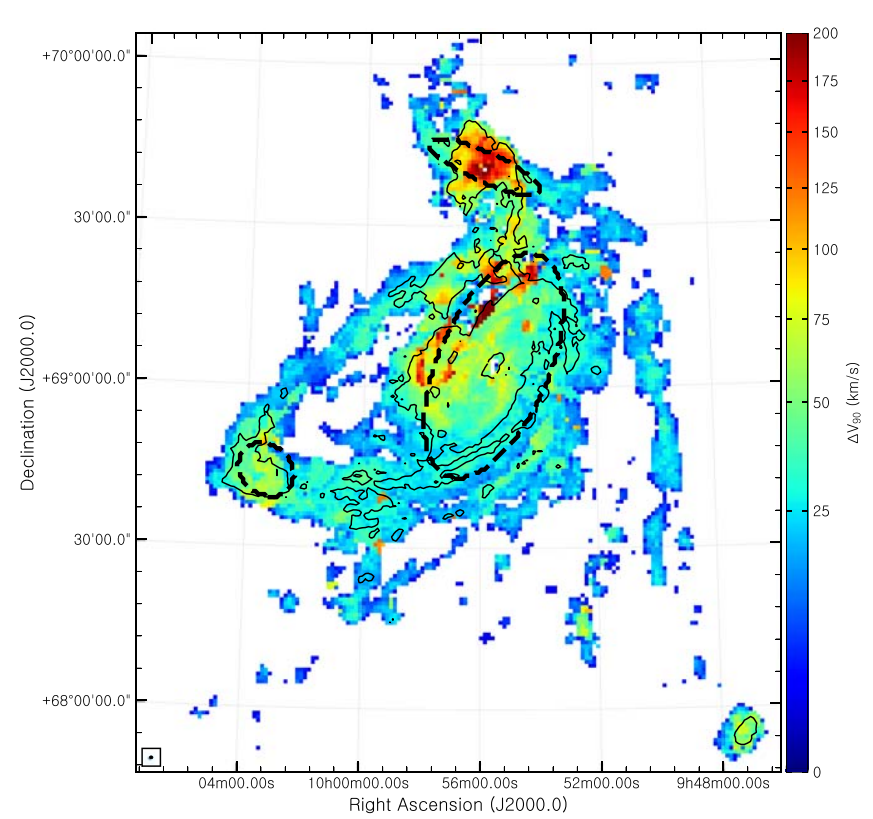

Figure 16. Map of $\Delta V_{90}$. The color map uses a square-root stretch. The thin black contour indicates the $2 \times 10^{20} \mathrm{~cm}^{-2}$ level. The black dashed ellipses indicate the optical disks, defined here as having a radius of $2 R_{25}$ where the optical positions, sizes, and orientation parameters have been taken from HyperLEDA.

factor two lower than the observed peak in the distribution for higher-redshift DLAs as found by Prochaska et al. (2008). They use the Yun et al. (1993a) H I data, along with VLA archival data, to determine the distribution of $\Delta V_{90}$ in M82 and find values similar to those found in DLAs. They conclude that superwinds and tidal features can to a large extent explain the higher $\Delta V_{90}$ values in DLAs. The presence of tidal features and outflows in the M81 triplet means it could be considered a local example of such high-redshift objects. Our observations cover the full spatial as well as spectral extent of the triplet, and are thus suitable to derive the M81 triplet velocity width distribution function which we can directly compare with the Zwaan et al. (2008) and DLA results.

We therefore revisit the $\Delta V_{90}$ analysis with our M81 triplet data set as well as a more recent DLA analysis by Neeleman et al. (2013). The latter study is based on high-resolution spectra of $100 \mathrm{HI}$-selected DLA systems, and includes a careful treatment of selection effects.

For the analysis we use the masked natural-weighted $\mathrm{C}+\mathrm{D}$ mosaic, but regridded to a the pixel size equal to $34^{\prime \prime}$, or the geometric mean of the beam size. This means each pixel is independent. For each spatial position we determine $\Delta V_{90}$ by constructing the normalized cumulative velocity profile, and measuring the velocities where the 5\% and 95\% levels occur. Note that for a Gaussian profile $\Delta V_{90}$ is equivalent to $3.28 \sigma$ where $\sigma$ is the dispersion.

Figure 16 shows the $\Delta V_{90}$ map of the M81 triplet. It shows similar features as the second-moment map in Figure 5, with the highest values also here occurring in and around M82 and the interface between M81 and M82. Typical values in the southern part of the triplet are $\sim 20-40 \mathrm{~km} \mathrm{~s}^{-1}$, while in the northern part these increase to $\sim 160-200 \mathrm{~km} \mathrm{~s}^{-1}$. As discussed in Section 3.1, these higher values are due to a combination of genuinely high-velocity spreads (due to outflows) and multiple components along the line of sight. 
A difference map of the $\Delta V_{90}$ and the second-moment maps (assuming that $\Delta V_{90}=3.28 \sigma$ ) has a median value of $0.1 \mathrm{~km} \mathrm{~s}^{-1}$. The only large differences occur in the regions with extreme velocity dispersions $\left(\gtrsim 70 \mathrm{~km} \mathrm{~s}^{-1}\right)$. In the southern disk of M81, where no such extreme dispersions are found, the average difference between the maps is $\sim-0.1 \pm 0.7 \mathrm{~km} \mathrm{~s}^{-1}$, showing that this analysis is largely independent of the way the velocity width is defined. We will return to this in Section 4.3.

Also shown in Figure 16 is the $2 \times 10^{20} \mathrm{~cm}^{-2}$ column density contour, which encloses the areas of the system that would give rise to DLA absorption. We also indicate the approximate areas of the optical disks (defined here as having a maximum radius of $2 R_{25}$ ) of the three main galaxies, using the optical positions and orientation parameters from HyperLEDA. We use $R_{25}=10^{\prime} \cdot 9$ for M81, 5!5 for M82, and 2!6 for NGC 3077.

Note that the majority of the DLA area (i.e., column density higher than $2 \times 10^{20} \mathrm{~cm}^{-2}$ ) occurs within the optical disks. Only a relatively small fraction, mainly between M81 and M82, and in the M81-NGC 3077 bridge, is found in the tidal features outside the disks.

Figure 17 shows the histogram of $\Delta V_{90}$ values as found within the $2 \times 10^{20} \mathrm{~cm}^{-2}$ contour in Figure 16. Also shown are the $\Delta V_{90}$ distribution as found by Zwaan et al. (2008) for the THINGS galaxies, and the measured values for DLA absorbers as presented in Prochaska et al. (2008), as well as the more recent measurements from Neeleman et al. (2013). The M81 triplet is characterized by a sharp peak and a long tail toward higher velocities. The peak occurs at $\sim 30 \mathrm{~km} \mathrm{~s}^{-1}$, identical to what was found for the THINGS galaxies. This reflects the fact that in terms of area, the triplet is dominated by the M81 disk, a rotationally supported disk just like the THINGS galaxies. The Neeleman et al. (2013) DLA values also strongly peak at $\sim 30 \mathrm{~km} \mathrm{~s}^{-1}$. They suggest that this peak corresponds to the "low-cool" part of the DLA population. These DLAs are thought to reside in smaller dark matter halos, where infalling gas is not shock-heated ("cold accretion") resulting in the formation of neutral gas disks (Wolfe et al. 2008). This is consistent with the current properties of M81 and the other THINGS galaxies.

The tail toward higher velocities is mainly caused by the high $\Delta V_{90}$ values found near M82. These values cover a similar range in high $\Delta V_{90}$ values as found for the DLAs. We can illustrate this in more detail by looking at the $\Delta V_{90}$ distribution as a function of environment. We define three disk environments using the definitions of the optical disks of the three major galaxies as shown in Figure 16, and select the high column density areas within these disks. The other high column density material outside these disks we define as being in a field or intra-disk environment. The $\Delta V_{90}$ histograms of each of these four environments are shown in Figure 17.

The M81 and the field distributions are similar at low velocities. The high-velocity tail of the field distribution is due to gas outside the optical disk along the minor axis of M82 with intrinsically higher dispersions, and gas in the M81-M82 interface with to multiple components along the line of sight (see Section 3.1). It is interesting that the same origin for the high $\Delta V_{90}$ was recently also found in results from numerical simulations (Bird et al. 2015).

The range in values found for NGC 3077 is similar to that for M81, though the peak of the NGC 3077 distribution occurs at $\sim 60 \mathrm{~km} \mathrm{~s}^{-1}$. This is mostly due the fact that the NGC 3077

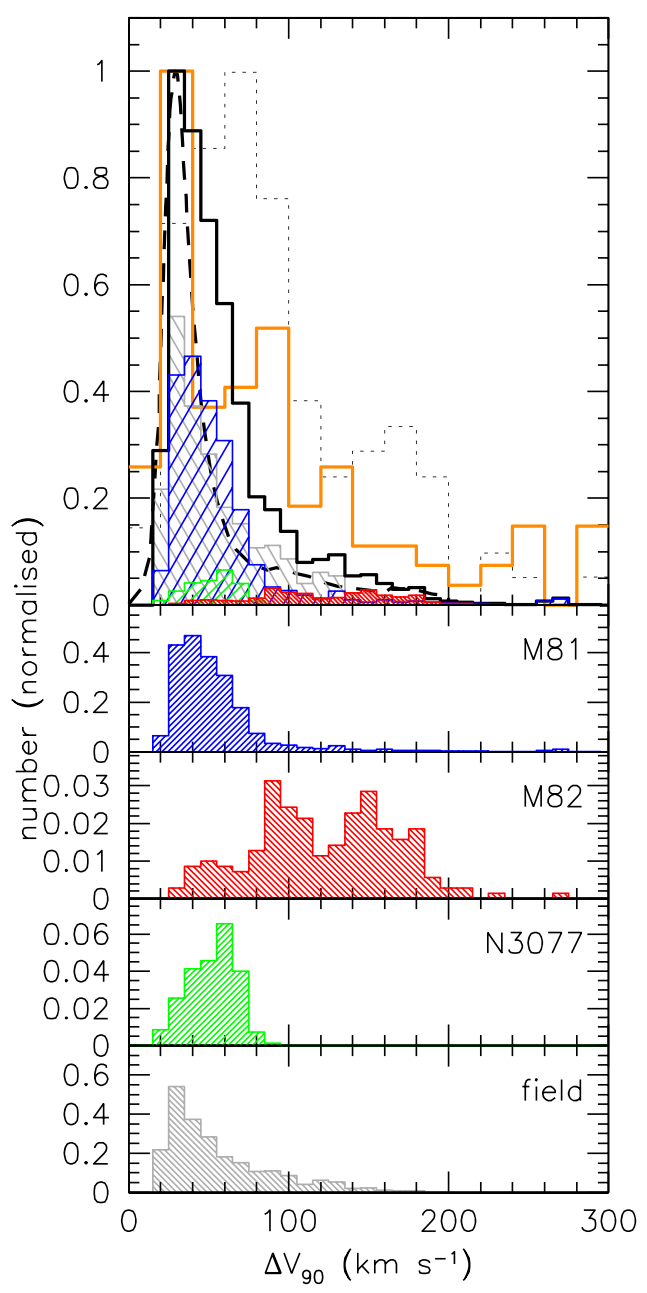

Figure 17. Histograms of $\Delta V_{90}$. The top panel shows the distribution for the M81 triplet selecting only column densities above $2 \times 10^{20} \mathrm{~cm}^{-2}$ (thick black full lines), a sample of DLA absorbers from Prochaska et al. (2008) (thin black dotted lines), a recent DLA sample from Neeleman et al. (2013) (orange full lines), and the THINGS sample (Zwaan et al. 2008, thick black dashed lines). The hatched histograms indicate the four environments found in the triplet. Blue: optical disk of M81 within $2 R_{25}$; red: M82 optical disk within $2 R_{25}$; green: optical disk of NGC 3077 within $2 R_{25}$; gray: field, i.e., any part of the triplet not included in the optical disks. The four bottom panels show zoom-ins on each of the four environment histograms. The histograms are created using maps where the pixel size is equal to the beam size, so that pixels are independent.

"disk" as indicated in Figure 16 encompasses part of the NGC 3077-M81 bridge, where dispersions are higher due to the presence of multiple components

M82 exhibits a much larger range in $\Delta V_{90}$ values which mostly reflect intrinsically high dispersions. It covers the same velocity range as the DLAs.

Note that as M82 can be regarded as an edge-on disk, some of the high $\Delta V_{90}$ values found there can be due to rotational broadening of the emission line. In a rotating disk, this effect will, however, only be important for lines of sight close to the (projected) center of the galaxy. Figure 16 shows that the area with high- $\Delta V_{90}$ values is much larger, and also extends further vertically than radially. Close inspection of Figure 17 shows that for $140 \mathrm{~km} \mathrm{~s}^{-1}<\Delta V_{90}<200 \mathrm{~km} \mathrm{~s}^{-1}$, only about half of the high $\Delta V_{90}$ values are found in the M82 environment as defined above. The other half occur outside this environment, but still in the general vicinity of M82. We conclude that the 


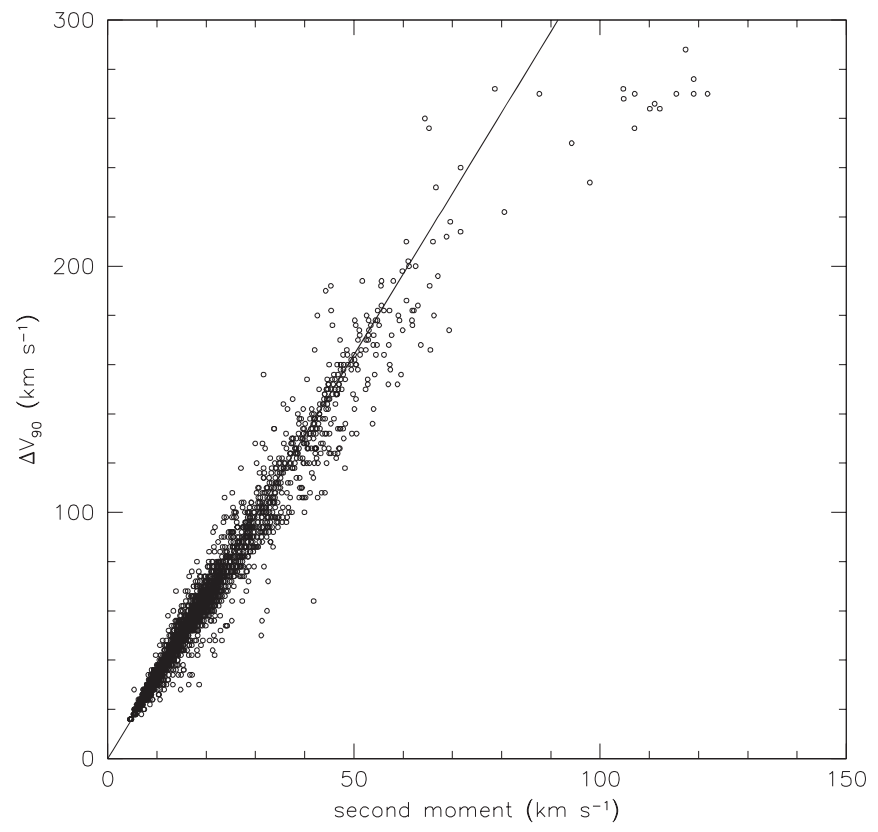

Figure 18. Plot of $\Delta V_{90}$ against the corresponding second-moment value. The line indicates the relation $\Delta V_{90}=3.28 \sigma$ expected for a Gaussian profile, where $\sigma$ is the dispersion (second moment) of the profile.

majority of high $\Delta V_{90}$ values found near M82 are associated with outflows, with rotational broadening playing a less important role.

Figure 17 thus shows that the M81 triplet can reproduce most of the velocity widths found in DLAs. The only exceptions are velocity widths $\gtrsim 200 \mathrm{~km} \mathrm{~s}^{-1}$, which are very rare in the triplet, but which do occur in DLAs. Nevertheless, we can ask the question as to how far we can reproduce the observed DLA $\Delta V_{90}$ distribution using only M81-like and M82-like galaxies. Restricting ourselves to $\Delta V_{90}<200 \mathrm{~km} \mathrm{~s}^{-1}$, we find that an increase of a factor between 2 and 5 of the number of high $\Delta V_{90}$ values, as found near M82, gives a distribution close to that of the Neeleman et al. (2013) DLA distribution.

\subsection{Velocity Dispersion and Column Density}

Focusing more on the properties of the M81 triplet itself, we can ask what the distribution of velocity dispersions and column densities is in the four disk/field environments defined above. We use velocity dispersions here, rather than $\Delta V_{90}$ values, as the former are easier to determine and can be directly related to literature values. Also, as noted above, for a Gaussian profile $\Delta V_{90}=3.28 \sigma$. In our data set, the majority of the profiles are (close to) Gaussian and the two types of velocity width can be directly intercompared. This is illustrated in Figure 18. The dozen points with second-moment values $>100 \mathrm{~km} \mathrm{~s}^{-1}$ correspond to the high-dispersion ridge already discussed in Section 2.5 , where the high values are caused by distinct, multiple, and widely separated components. These non-Gaussian profiles obviously have a different impact on the second-moment and $\Delta V_{90}$ values.

We now also include column densities below $2 \times 10^{20} \mathrm{~cm}^{-2}$, allowing us to more fully address questions such as whether the $\mathrm{HI}$ velocity dispersion depends on column density, and whether tidal material can be identified based purely on the column density and/or velocity dispersion.

We use the zeroth- and second-moment natural-weighted $\mathrm{C}+\mathrm{D}$ array maps presented in Figures 3 and 5 but, as above, regridded to a pixel size of $34^{\prime \prime}$ so that one pixel is approximately equal to the beam size and yields an independent measurement. We furthermore subdivide the disks in an inner disk $\left(0<R<R_{25}\right)$ and an outer disk $\left(R_{25}<R<2 R_{25}\right)$.

Figure 19 shows histograms of the column densities and velocity dispersions in the entire $3^{\circ} \times 3^{\circ}$ field. We see that the velocity dispersion histogram peaks close to the spectral resolution limit of the datacube. The median value of the velocity dispersion is $8.6 \mathrm{~km} \mathrm{~s}^{-1}$. M82 lacks the low-velocity dispersion peak, while NGC 3077 only shows relatively low dispersions.

In terms of column densities, we see that the field on average shows lower column densities than the galaxies, which of course is not unexpected from inspection of the moment maps. The column density distributions of M81, M82 and NGC 3077 are similar in shape.

We can split these distributions up further and investigate differences between inner and outer disk. For M81 there are a large number of high dispersion values in the outer disk compared to the inner disk. These are not physical, but indicate the presence of multiple components, as already discussed. In M82 the velocity dispersions of the inner disk are higher than in the outer disk, just as in NGC 3077. Note that the spread and average values for M82 are significantly higher than those of NGC 3077. The column density histograms show that for M81 and M82 the highest column densities are found in the inner disk. This is not the case for NGC 3077, which is due to the offset of the main H I component from the optical center of the galaxy.

Finally we compare the column densities and velocity dispersions, see Figure 20. The top-left panel compares the column density-velocity dispersion distribution of the entire field with those of the inner disks of the three main galaxies. The bulk of the $\mathrm{H}$ I has a column density of $\sim 10^{20}$ and a velocity dispersion of $\sim 7 \mathrm{~km} \mathrm{~s}^{-1}$, but there is a clear trend of velocity dispersion with column density. The three galaxies all sit at the upper tip of this distribution, but each occupy a different average position. The bulk of the $\mathrm{HI}$ in the inner disk of M81 has a column density of $\sim 10^{21} \mathrm{~cm}^{-2}$ and a velocity dispersion of $\sim 14 \mathrm{~km} \mathrm{~s}^{-1}$. The inner disk of M82 sits at a similar column density level, but has a higher velocity dispersion of $\sim 45 \mathrm{~km} \mathrm{~s}^{-1}$, though with a large spread. NGC 3077 sits at an intermediate position with an average column density of around $3 \times 10^{20} \mathrm{~cm}^{-2}$ and a velocity dispersion of $\sim 20 \mathrm{~km} \mathrm{~s}^{-1}$.

The other three panels in Figure 20 compare the inner and outer disks of the three galaxies with the total distribution. The outer disk of M81 has, not unexpectedly, a lower velocity dispersion and column density than the inner disk, but note the significant number of points that have very high velocity dispersions. Comparing this with the typical column densities of the M82 high dispersion locations, we can conclude that high velocity dispersions at column densities below a few times $10^{20} \mathrm{~cm}^{-2}$ indicate with a high probability that these are due to multiple gas components.

M82 also shows a clear column density-velocity dispersion relation, though we find a significant number of outer disk points with high velocity dispersions. This is, to a large extent, due the gas present along the minor axis (i.e., above and below the disk), which makes distinction between inner and outer disk somewhat artifical.

NGC 3077 shows no clear trend, and no real distinction between inner and outer disk. In this case, interpretation is complicated further by the offset between the H I and optical components. 

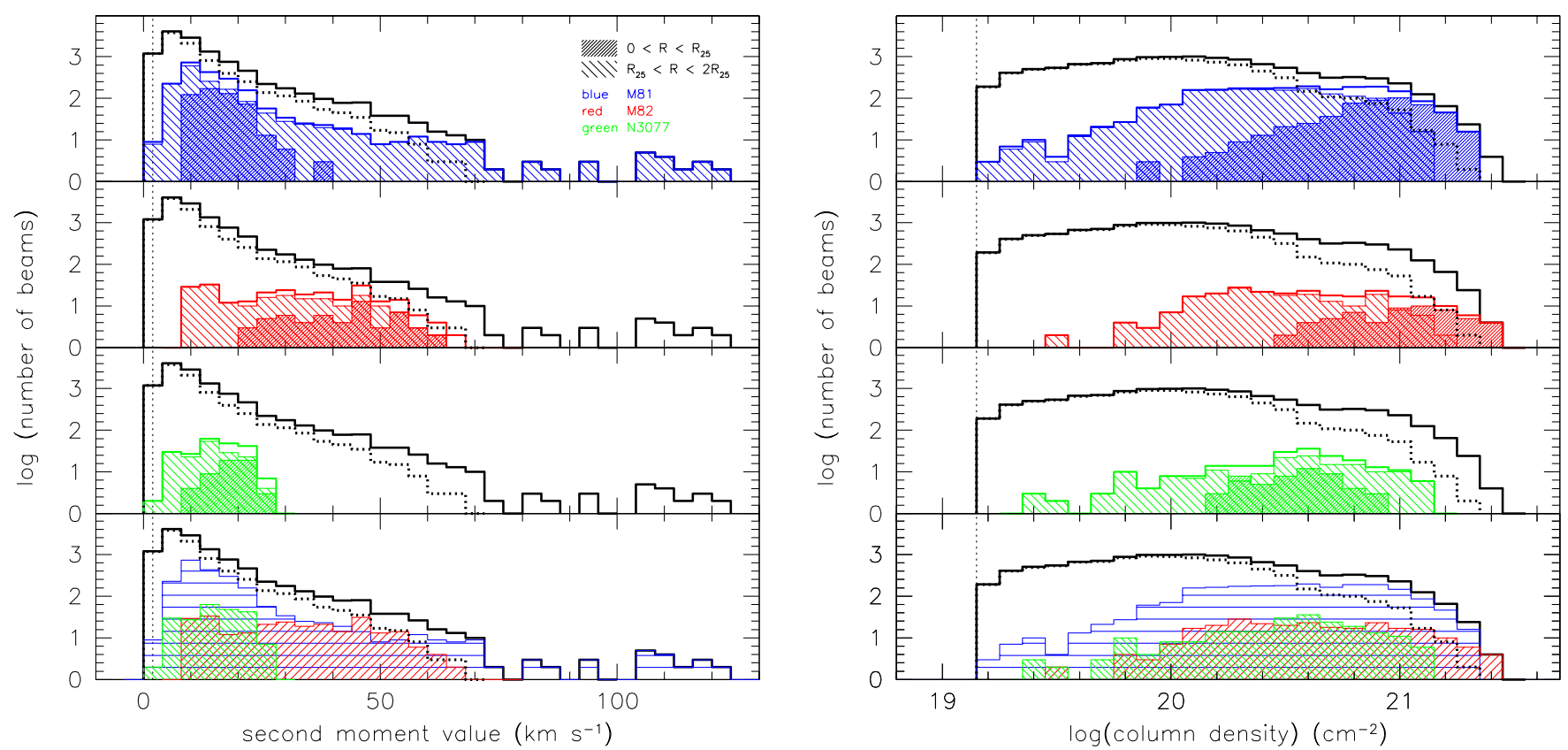

Figure 19. Histograms of the velocity dispersions (left panel) and column densities (right). In all sub-panels the full, thick histogram represents the entire observed area; the dotted histogram shows the field environment; the blue histogram in the top sub-panel shows the M81 disk environment; the red histogram in the second subpanel from the top shows the M82 environment and the green histogram in the third sub-panel from the top shows the NGC 3077 environment. In these three subpanels, single hatched histograms indicate the outer disk, cross-hatched histograms the inner disk. The bottom sub-panels compare the three environments. The blue horizontally hatched histogram represents the M81 environment, the red $45^{\circ}$-hatched histogram the M82 environment, and the green $-45^{\circ}$-hatched histogram the NGC 3077 environment. The vertical dotted lines in all sub-panels indicate the respective velocity dispersion and column density limits of the data.

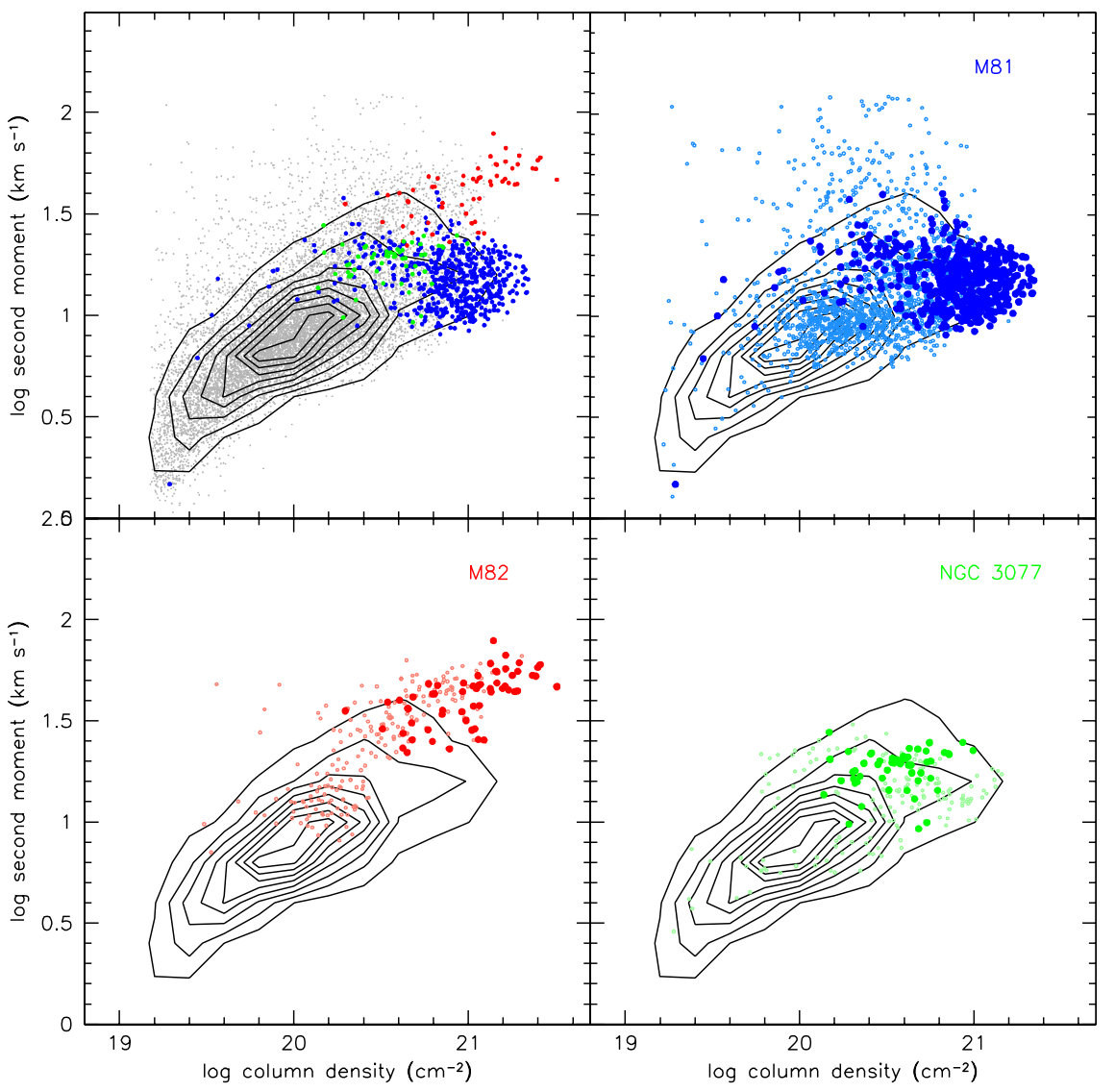

Figure 20. Pixel-pixel comparisons of column densities and velocity dispersions. The top-left panel compares the distribution of the entire mosaic (gray points, with black density contours overplotted), and the inner disks of M81 (blue), M82 (red), and NGC 3077 (green). Contour levels are equally spaced, running from 100 to 800. The other three panels compare the inner (large filled symbols) and outer disks (small open symbols) of the three main galaxies with the total distribution (contours). Top-right: M81 inner disk (blue) and outer disk (light-blue). Bottom-left: M82 inner disk (red) and outer disk (light-red). Bottom-right: NGC 3077 inner disk (green) and outer disk (light green). 
In summary, the three inner disks of the triplet galaxies occupy distinct positions in column density-velocity dispersion space. High velocity dispersions at high column densities most likely reflect intrinsically high dispersions, while similar dispersions at low column densities are due to the presence of multiple components at different velocities.

\section{Summary}

We have presented a new, high-resolution, 105-pointing $\mathrm{H} \mathrm{I}$ mosaic of $3^{\circ} \times 3^{\circ}$ centered on the M81 triplet, M81, M82, and NGC 3077, obtained with the VLA in its C- and D-configurations. This is the first radio synthesis data set that maps the entire volume of the triplet at high spatial and spectral resolution. These data can serve as input for further sophisticated modeling of the interaction and evolution of the triplet (e.g., Oehm et al. 2017).

Our main results are summarized as follows.

1. We do not find a large population of free-floating $\mathrm{HI}$ clouds down to an $\mathrm{HI}$ mass limit of $\sim 10^{4} \mathrm{M}_{\odot}$. While small clouds and H I complexes are detected they only occur close (spatially and spectrally) to the main $\mathrm{H}$ I tidal features of the triplet, suggesting they are all debris of the interaction that shaped the triplet. A detailed investigation of the H I masses of these clouds show that they are likely embedded in extended low column density tidal features.

2. Comparison with a sensitive GBT H I mosaic of the same area by Chynoweth et al. (2008) shows that the VLA mosaic has detected most of the $\mathrm{H} \mathrm{I}$ in the southern part of the mosaic (i.e., the southern part of M81 and NGC 3077). In the northern part of the mosaic (M82 and the the M81-M82 transition region) the GBT has detected a significant excess of flux most likely associated with M82. This probably indicates the presence of a low column density H I component associated with the M82 outflows.

3. Using additional data we show that low column density features detected by the GBT beyond the SE edge of our VLA mosaic are resolved into clouds. In turn, we detect a small H I cloud beyond the extent of the GBT mosaic, suggesting that a low column density $\mathrm{H}$ I tail resulting from the interaction may extend further SE beyond the areas mapped by the VLA and GBT.

4. A comparison of the velocity widths and H I masses of these clouds seems consistent with them being dominated by dark matter. Their properties are, in that regard, very similar to those of UHVCs or the smallest gas-rich dwarf galaxies. However, given their association with tidal features, it is more likely that the velocity widths should not be interpreted in terms of gravitational support. It is possible that these clouds will eventually evolve into tidal dwarf galaxies.

5. We compare the observed HI column densities with a Subaru Suprime-Cam map of the resolved young stellar population of the triplet. The majority of the $\mathrm{OB}$ star distribution is found within the $6 \times 10^{20} \mathrm{~cm}^{-2}$ contour. After taking projection effects into account, this is consistent with theoretical predictions for the star formation threshold surface density value.

6. We derive the distribution of $\Delta V_{90}$ of the $\mathrm{H}$ I profiles and compare these with that observed for DLAs to investigate whether the triplet can be regarded as a local version of the high- $z$ objects that cause the DLA absorption. We find that the peaks of the distributions coincide at low $\Delta V_{90}$ values, consistent with the interpretation that the low $\Delta V_{90}$ values occur in objects that will evolve in neutral gas disks. High $\Delta V_{90}$ values are found around M82, and these cover the entire range in $\Delta V_{90}$ found in DLAs up to $200 \mathrm{~km} \mathrm{~s}^{-1}$. This is consistent with high $\Delta V_{90}$ values being caused by feedback, outflows, or multiple components along the line of sight. For the triplet to also reproduce the relative fraction of high versus low $\Delta V_{90}$ values found in DLAs, the frequency of the values found near M82 needs to be increased by a factor of 2-5, presumably indicating that in DLAs the relative importance of feedback and outflow effects is somewhat more important than in the triplet.

We thank the referee for constructive comments that have made a significant difference to this paper.

The National Radio Astronomy Observatory is a facility of the National Science Foundation operated under cooperative agreement by Associated Universities, Inc.

Funding for the Sloan Digital Sky Survey IV has been provided by the Alfred P. Sloan Foundation, the U.S. Department of Energy Office of Science, and the Participating Institutions. SDSS-IV acknowledges support and resources from the Center for High-Performance Computing at the University of Utah. The SDSS web site is www.sdss.org.

SDSS-IV is managed by the Astrophysical Research Consortium for the Participating Institutions of the SDSS Collaboration including the Brazilian Participation Group, the Carnegie Institution for Science, Carnegie Mellon University, the Chilean Participation Group, the French Participation Group, Harvard-Smithsonian Center for Astrophysics, Instituto de Astrofísica de Canarias, The Johns Hopkins University, Kavli Institute for the Physics and Mathematics of the Universe (IPMU)/University of Tokyo, Lawrence Berkeley National Laboratory, Leibniz Institut für Astrophysik Potsdam (AIP), Max-Planck-Institut für Astronomie (MPIA Heidelberg), Max-Planck-Institut für Astrophysik (MPA Garching), MaxPlanck-Institut für Extraterrestrische Physik (MPE), National Astronomical Observatories of China, New Mexico State University, New York University, University of Notre Dame, Observatário Nacional/MCTI, The Ohio State University, Pennsylvania State University, Shanghai Astronomical Observatory, United Kingdom Participation Group, Universidad Nacional Autónoma de México, University of Arizona, University of Colorado Boulder, University of Oxford, University of Portsmouth, University of Utah, University of Virginia, University of Washington, University of Wisconsin, Vanderbilt University, and Yale University.

\section{ORCID iDs}

Fabian Walter (iD https://orcid.org/0000-0003-4793-7880 Annette M. N. Ferguson (iD https://orcid.org/0000-00017934-1278

Edouard J. Bernard (10 https://orcid.org/0000-0002-8722-225X Adam K. Leroy (i) https://orcid.org/0000-0002-2545-1700

Jürgen Ott (i) https://orcid.org/0000-0001-8224-1956

Laura K. Zschaechner (iD https://orcid.org/0000-00029919-8672

Martin A. Zwaan (iD https://orcid.org/0000-0003-0101-1804

Min S. Yun (10) https://orcid.org/0000-0001-7095-7543 


\section{References}

Adams, E. A. K., Oosterloo, T. A., Cannon, J. M., Giovanelli, R., \& Haynes, M. P. 2016, A\&A, 596, A117

Appleton, P. N., Davies, R. D., \& Stephenson, R. J. 1981, MNRAS, 195, 327 Appleton, P. N., \& van der Hulst, J. M. 1988, MNRAS, 234, 957

Barker, M. K., Ferguson, A. M. N., Irwin, M., Arimoto, N., \& Jablonka, P. 2009, AJ, 138, 1469

Barnes, L. A., \& Haehnelt, M. G. 2014, MNRAS, 440, 2313

Bernard, E. J., Ferguson, A. M. N., Barker, M. K., et al. 2012, MNRAS, 426, 3490

Bernstein-Cooper, E. Z., Cannon, J. M., Elson, E. C., et al. 2014, AJ, 148, 35

Bigiel, F., Leroy, A., Seibert, M., et al. 2010, ApJL, 720, L31

Bigiel, F., Leroy, A., Walter, F., et al. 2008, AJ, 136, 2846

Bird, S., Haehnelt, M., Neeleman, M., et al. 2015, MNRAS, 447, 1834

Brouillet, N., Baudry, A., Combes, F., Kaufman, M., \& Bash, F. 1991, A\&A, 242,35

Chynoweth, K. M., Langston, G. I., \& Holley-Bockelmann, K. 2011, AJ, 141, 9

Chynoweth, K. M., Langston, G. I., Yun, M. S., et al. 2008, AJ, 135, 1983

Davidge, T. J. 2008, ApJL, 678, L85

de Blok, W. J. G., Walter, F., Brinks, E., et al. 2008, AJ, 136, 2648

de Vaucouleurs, G., de Vaucouleurs, A., Corwin, H. G., et al. 1991, Third Reference Catalogue of Bright Galaxies. Vol. I, Vol. II, Vol. III (New York: Springer)

Ferguson, A. M. N., Irwin, M. J., Ibata, R. A., Lewis, G. F., \& Tanvir, N. R. 2002, AJ, 124, 1452

Ferguson, A. M. N., Wyse, R. F. G., Gallagher, J. S., \& Hunter, D. A. 1998, ApJL, 506, L19

Gil de Paz, A., Boissier, S., Madore, B. F., et al. 2007, ApJS, 173, 185

Greenawalt, B., Walterbos, R. A. M., Thilker, D., \& Hoopes, C. G. 1998, ApJ, 506,135

Hoopes, C. G., Heckman, T. M., Strickland, D. K., et al. 2005, ApJL, 619, L99

Hunter, D. A., Elmegreen, B. G., \& Baker, A. L. 1998, ApJ, 493, 595

Ianjamasimanana, R., de Blok, W. J. G., \& Heald, G. H. 2017, AJ, 153, 213

Jörsäter, S., \& van Moorsel, G. A. 1995, AJ, 110, 2037

Karachentsev, I. D., Dolphin, A. E., Geisler, D., et al. 2002, A\&A, 383, 125

Karachentsev, I. D., Karachentseva, V. E., Huchtmeier, W. K., \& Makarov, D. I. 2004, AJ, 127, 2031

Kauffmann, G., White, S. D. M., \& Guiderdoni, B. 1993, MNRAS, 264, 201

Kennicutt, R. C., \& Evans, N. J. 2012, ARA\&A, 50, 531

Kennicutt, R. C., Jr. 1989, ApJ, 344, 685

Leroy, A. K., Walter, F., Brinks, E., et al. 2008, AJ, 136, 2782

Leroy, A. K., Walter, F., Martini, P., et al. 2015, ApJ, 814, 83

Martini, P., Leroy, A. K., Mangum, J. G., et al. 2018, arXiv:1802.04359

McMullin, J. P., Waters, B., Schiebel, D., Young, W., \& Golap, K. 2007, in ASP Conf. Ser. 376, Astronomical Data Analysis Software and Systems XVI, ed. R. A. Shaw, F. Hill, \& D. J. Bell (San Francisco, CA: ASP), 127

McQuinn, K. B. W., Skillman, E. D., Dolphin, A., et al. 2015, ApJ, 812, 158

Meier, D. S., Turner, J. L., \& Beck, S. C. 2001, AJ, 122, 1770

Miyazaki, S., Komiyama, Y., Sekiguchi, M., et al. 2002, PASJ, 54, 833

Mouhcine, M., \& Ibata, R. 2009, MNRAS, 399, 737

Neeleman, M., Wolfe, A. M., Prochaska, J. X., \& Rafelski, M. 2013, ApJ, 769,54
Nilson, P. 1973, Uppsala General Catalogue of Galaxies, Uppsala Astr. Obs. Annaler, Band 6

Oehm, W., Thies, I., \& Kroupa, P. 2017, MNRAS, 467, 273

Offringa, A. R., de Bruyn, A. G., Biehl, M., et al. 2010, MNRAS, 405, 155

Okamoto, S., Arimoto, N., Ferguson, A. M. N., et al. 2015, ApJL, 809, L1

Pontzen, A., Governato, F., Pettini, M., et al. 2008, MNRAS, 390, 1349

Prochaska, J. X., Chen, H.-W., Wolfe, A. M., Dessauges-Zavadsky, M., \& Bloom, J. S. 2008, ApJ, 672, 59

Prochaska, J. X., Ryan-Weber, E., \& Staveley-Smith, L. 2002, PASP, 114, 1197

Rahmati, A., \& Schaye, J. 2014, MNRAS, 438, 529

Rots, A. H. 1975, A\&A, 45, 43

Sánchez-Gallego, J. R., Knapen, J. H., Heiner, J. S., et al. 2011, A\&A, 527, A16

Sault, R. J., Teuben, P. J., \& Wright, M. C. H. 1995, in ASP Conf. Ser. 77, Astronomical Data Analysis Software and Systems IV, ed. R. Shaw, H. E. Payne, \& J. J. E. Hayes (San Francisco, CA: ASP), 433

Schaye, J. 2001, ApJL, 559, L1

Schaye, J. 2004, ApJ, 609, 667

Schlafly, E. F., \& Finkbeiner, D. P. 2011, ApJ, 737, 103

Schlegel, D. J., Finkbeiner, D. P., \& Davis, M. 1998, ApJ, 500, 525

Serra, P., Oosterloo, T., Morganti, R., et al. 2012, MNRAS, 422, 1835

Serra, P., Westmeier, T., Giese, N., et al. 2015, MNRAS, 448, 1922

Skillman, E. D. 1987, in Star Formation in Galaxies, (NASA Conf. Pub. CP2466), ed. C. J. Londsdale Persson, 263

Stetson, P. B. 1994, PASP, 106, 250

Sun, W.-H., Zhou, X., Chen, W.-P., et al. 2005, ApJL, 630, L133

Tescari, E., Viel, M., Tornatore, L., \& Borgani, S. 2009, MNRAS, 397, 411

van der Hulst, J. M. 1979, A\&A, 75, 97

Walter, F., Brinks, E., de Blok, W. J. G., et al. 2008, AJ, 136, 2563

Walter, F., \& Heithausen, A. 1999, ApJL, 519, L69

Walter, F., Martin, C. L., \& Ott, J. 2006, AJ, 132, 2289

Walter, F., Sandstrom, K., Aniano, G., et al. 2011, ApJL, 726, L11

Walter, F., Weiss, A., Martin, C., \& Scoville, N. 2002a, AJ, 123, 225

Walter, F., Weiss, A., \& Scoville, N. 2002b, ApJL, 580, L21

Wolfe, A. M., Gawiser, E., \& Prochaska, J. X. 2005, ARA\&A, 43, 861

Wolfe, A. M., Lanzetta, K. M., Foltz, C. B., \& Chaffee, F. H. 1995, ApJ, 454,698

Wolfe, A. M., Prochaska, J. X., Jorgenson, R. A., \& Rafelski, M. 2008, ApJ, 681,881

Wolfe, A. M., Turnshek, D. A., Smith, H. E., \& Cohen, R. D. 1986, ApJS, 61,249

Yun, M. S. 1999, in Proc. IAU Symp. 186, Galaxy Interactions at Low and High Redshift, ed. J. E. Barnes \& D. B. Sanders (Dordrecht: Kluwer), 81

Yun, M. S., Ho, P. T. P., Brouillet, N., \& Lo, K. Y. 1993a, in NASA Ames Research Center, The Evolution of Galaxies and their Environment, ed. J. M. Shull \& H. A. Thronson, 253

Yun, M. S., Ho, P. T. P., \& Lo, K. Y. 1993b, ApJL, 411, L17

Yun, M. S., Ho, P. T. P., \& Lo, K. Y. 1994, Natur, 372, 530

Yun, M. S., Ho, P. T. P., \& Lo, K. Y. 2000, in ASP Conf. Proc. 217, Imaging at Radio through Submillimeter Wavelengths, ed. J. G. Mangum \& S. J. E. Radford (San Francisco, CA: ASP), 374

Zwaan, M., Walter, F., Ryan-Weber, E., et al. 2008, AJ, 136, 2886 